\title{
22. GRAIN SIZE RESULTS AND COMPOSITION OF THE SAND FRACTION, LEG 6
}

\author{
A. C. Pimm, Scripps Institution of Oceanography, La Jolla, California
}

\section{GRAIN SIZE RESULTS}

The complete grain size results are given in Table 1, the triangle diagrams for each site as Figure 1 and down hole plots as Figure 2. Cumulative curves are given in Chapter 27.

\section{Site 44}

Sediment throughout Hole 44.0 is nannoplankton chalk ooze with a grain size distribution predominantly in the clayey silt range. The moderately high silt content is due to an abundance of nannoplankton. The sand content is low nearly everywhere, being almost totally comprised of foraminifera. Rarely the sand content is moderate (Core 3-CC) or high (Core 5-0) due to an abundance of chert fragments (produced as artifacts of drilling) in addition to foraminifera.

\section{Site 45}

In Hole 45.1, Core 1, pelagic brown clays fall in the clay-size range, but interbedded volcanic ashes are of clayey silt-size.

\section{Site 46}

Pelagic brown clays in Core 1 fall in the clay-size range, but admixtures of ash and chert in places give the sediment a clayey silt or sandy clay classification.

\section{Site 47}

At this site three holes were drilled, all of which penetrated a similar sequence of nannoplankton chalk ooze. The Neogene sediments fall in the silty clay, or rarely clay (part of Core 5), size range. These younger sediments rest unconformably on Paleogene and Cretaceous sediments which show an increase in the silt fractionnoticeably in Cores 7 and 8 which are of clayey silt size.

\section{Site 48}

The two holes drilled at Site 48 both recovered nannoplankton chalk ooze which falls in the silty clay-size range.

\section{Site 49}

Two holes drilled at Site 49 recovered interbedded brown pelagic clay and nannoplankton chalk ooze. Both sediment types fall mainly in the clay and silty clay range. The silt content in the brown clay is due to an admixture of chert, ash and chalk fragments. The silt content of the chalk ooze is unusually low and possibly due to the small size of the nannoplankton in the sediment.

\section{Site $\mathbf{5 0}$}

Pelagic brown clay and nannoplankton chalk ooze were recovered from two holes drilled at this site. The brown clay falls in the clay-size range, but in Sections 1 and 3 of Core 2, Hole 50.1, the sediment is of clayey silt size due to a considerable admixture of volcanic ash. The chalk ooze falls in the silty clay-size range, except Hole 50.0, Core 2, Section 6, where common chert fragments raise the sand fraction to over 20 per cent.

\section{Site 51}

Pelagic brown clay from Holes 51.0 and 51.1 has a very low silt content (usually less than 10 per cent). In Core 1 in Hole 51.1 an admixture of volcanic ash raises the silt content from about 25 to 45 per cent.

\section{Site 52}

Pelagic brown clay in Hole 52.0 contains varying amounts of volcanic ash and depending on this the grain size ranges from clay through silty clay to clayey silt. In Core 8, the sand fraction shows a noticeable increase due to common lithified ash and chert fragments.

\section{Site 53}

Hole 53.0 recovered volcanic ash mainly of clayey silt size, with some silt or sandy silt interbeds, in Cores 1 through 3 . In Core 4 the ash becomes finer and is mainly of clay size; this is probably due to extensive alteration of the volcanic glass in the sediment.

In Core 6 of Hole 53.0 "nodules" of friable limestone are of silty sand size, while the softer chalky matrix in which they occur is of silty clay size.

In Core 7 altered volcanic rock gave a sand-silt-clay grain size.

In Hole 53.1, brown clay in Core 1 is mainly of silty clay size. Volcanic ash in Core 2 is of sand or silty sand size in the upper part, but becomes finer lower down with the silt fraction predominating. Core 3 is similar with ash of sandy or clayey silt size. 
TABLE 1

Leg 6 Grain Size Results

\begin{tabular}{|c|c|c|c|c|c|c|c|}
\hline Hole & Core & Section & $\begin{array}{l}\text { Sampled } \\
\text { at (m) }\end{array}$ & $\begin{array}{l}\text { Sand } \\
(\%)\end{array}$ & $\begin{array}{l}\text { Silt } \\
(\%)\end{array}$ & $\begin{array}{l}\text { Clay } \\
(\%)\end{array}$ & Classification \\
\hline 44.0 & 1 & 1 & 145.0 & 3.7 & 59.6 & 36.6 & Clayey Silt \\
\hline 44.0 & 1 & 2 & 145.0 & 7.2 & 31.5 & 61.3 & Silty Clay \\
\hline 44.0 & 1 & 3 & 150.0 & 5.1 & 55.7 & 39.3 & Clayey Silt \\
\hline 44.0 & 1 & 3 & 145.0 & 3.6 & 55.3 & 41.1 & Clayey Silt \\
\hline 44.0 & 1 & 4 & 145.0 & 5.0 & 59.1 & 35.9 & Clayey Silt \\
\hline 44.0 & 2 & 1 & 145.0 & 0.6 & 63.0 & 36.4 & Clayey Silt \\
\hline 44.0 & 2 & 2 & 145.0 & 0.6 & 61.1 & 38.3 & Clayey Silt \\
\hline 44.0 & 2 & 3 & 145.0 & 0.8 & 58.9 & 40.3 & Clayey Silt \\
\hline 44.0 & 2 & 4 & 145.0 & 0.7 & 58.2 & 41.1 & Clayey Silt \\
\hline 44.0 & 2 & 5 & 145.0 & 0.8 & 60.4 & 38.8 & Clayey Silt \\
\hline 44.0 & 2 & $\mathrm{CC}$ & 0.0 & 0.4 & 62.8 & 36.9 & Clayey Silt \\
\hline 44.0 & 3 & 2 & 145.0 & 1.0 & 64.7 & 34.3 & Clayey Silt \\
\hline 44.0 & 3 & 3 & 145.0 & 0.5 & 72.6 & 26.9 & Clayey Silt \\
\hline 44.0 & 3 & 4 & 145.0 & 1.4 & 58.7 & 39.9 & Clayey Silt \\
\hline 44.0 & 3 & 5 & 145.0 & 8.1 & 54.1 & 37.8 & Clayey Silt \\
\hline 44.0 & 3 & $\mathrm{CC}$ & 0.0 & 21.5 & 45.5 & 32.9 & Sand-Silt-Clay \\
\hline 44.0 & 4 & 0 & 0.0 & 6.8 & 54.0 & 39.2 & Clayey Silt \\
\hline 44.0 & 4 & 2 & 145.0 & 3.7 & 56.7 & 39.6 & Clayey Silt \\
\hline 44.0 & 4 & 3 & 145.0 & 1.6 & 56.1 & 42.3 & Clayey Silt \\
\hline 44.0 & 4 & 4 & 145.0 & 3.4 & 60.9 & 35.7 & Clayey Silt \\
\hline 44.0 & 4 & 5 & 145.0 & 1.1 & 61.0 & 37.9 & Clayey Silt \\
\hline 44.0 & 4 & 6 & 145.0 & 12.5 & 52.2 & 35.3 & Clayey Silt \\
\hline 44.0 & 4 & $\mathrm{CC}$ & 0.0 & 6.7 & 57.5 & 35.7 & Clayey Silt \\
\hline 44.0 & 5 & 0 & 0.0 & 71.0 & 17.7 & 11.3 & Silty Sand \\
\hline 45.1 & 1 & 1 & 20.0 & 0.4 & 2.1 & 97.5 & Clay \\
\hline 45.1 & 1 & 2 & 103.0 & 0.3 & 12.8 & 86.9 & Clay \\
\hline 45.1 & 1 & 2 & 128.0 & 0.2 & 66.6 & 33.2 & Clayey Silt \\
\hline 45.1 & 1 & 3 & 38.0 & 0.4 & 63.5 & 36.1 & Clayey Silt \\
\hline 45.1 & 1 & 4 & 67.0 & 1.6 & 65.5 & 32.9 & Clayey Silt \\
\hline 45.1 & 1 & 5 & 3.0 & 3.1 & 63.5 & 33.4 & Clayey Silt \\
\hline 46.0 & 1 & 1 & 145.0 & 3.4 & 66.3 & 30.4 & Clayey Silt \\
\hline 46.0 & 1 & 2 & 145.0 & 3.4 & 18.8 & 77.8 & Clay \\
\hline 46.0 & 1 & 3 & 145.0 & 9.7 & 14.6 & 75.7 & Clay \\
\hline 46.0 & 1 & 4 & 145.0 & 4.0 & 17.7 & 78.4 & Clay \\
\hline 46.0 & 1 & 5 & 145.0 & 24.9 & 18.0 & 57.1 & Sandy Clay \\
\hline 46.0 & 1 & 6 & 145.0 & 3.5 & 16.9 & 79.6 & Clay \\
\hline 47.0 & 1 & 1 & 76.0 & 3.4 & 37.1 & 59.5 & Silty Clay \\
\hline 47.0 & 1 & 2 & 14.0 & 2.8 & 39.4 & 57.8 & Silty Clay \\
\hline 47.0 & 1 & 3 & 39.0 & 9.4 & 34.8 & 55.8 & Silty Clay \\
\hline 47.0 & 1 & 4 & 5.0 & 6.2 & 28.8 & 65.0 & Silty Clay \\
\hline 47.0 & 1 & 4 & 83.0 & 12.8 & 35.6 & 51.6 & Silty Clay \\
\hline 47.0 & 1 & 5 & 12.0 & 4.0 & 23.7 & 72.2 & Silty Clay \\
\hline 47.0 & 1 & 6 & 6.0 & 7.7 & 25.2 & 67.0 & Silty Clay \\
\hline
\end{tabular}


TABLE 1 - Continued

\begin{tabular}{|c|c|c|c|c|c|c|c|}
\hline Hole & Core & Section & $\begin{array}{l}\text { Sampled } \\
\text { at (m) }\end{array}$ & $\begin{array}{l}\text { Sand } \\
(\%)\end{array}$ & $\begin{array}{l}\text { Silt } \\
(\%)\end{array}$ & $\begin{array}{l}\text { Clay } \\
(\%)\end{array}$ & Classification \\
\hline 47.1 & 1 & 1 & 145.0 & 0.0 & 36.4 & 63.6 & Silty Clay \\
\hline 47.1 & 1 & 2 & 9.0 & 12.5 & 34.0 & 53.5 & Silty Clay \\
\hline 47.2 & 1 & 2 & 16.0 & 4.8 & 28.5 & 66.6 & Silty Clay \\
\hline 47.2 & 1 & 3 & 3.5 & 10.8 & 28.1 & 61.1 & Silty Clay \\
\hline 47.2 & 1 & 3 & 114.0 & 14.7 & 32.5 & 52.8 & Silty Clay \\
\hline 47.2 & 1 & 4 & 1.0 & 6.3 & 32.7 & 61.1 & Silty Clay \\
\hline 47.2 & 2 & 3 & 1.0 & 11.3 & 23.4 & 65.3 & Silty Clay \\
\hline 47.2 & 2 & 4 & 1.0 & 12.0 & 27.3 & 60.7 & Silty Clay \\
\hline 47.2 & 2 & 5 & 0.0 & 4.2 & 25.6 & 70.2 & Silty Clay \\
\hline 47.2 & 2 & 6 & 0.5 & 5.1 & 25.1 & 69.9 & Silty Clay \\
\hline 47.2 & 3 & 2 & 1.0 & 6.5 & 33.3 & 60.2 & Silty Clay \\
\hline 47.2 & 3 & 3 & 0.5 & 3.2 & 24.9 & 71.8 & Silty Clay \\
\hline 47.2 & 3 & 4 & 11.0 & 2.3 & 28.3 & 69.5 & Silty Clay \\
\hline 47.2 & 4 & 1 & 10.5 & 4.8 & 25.6 & 69.5 & Silty Clay \\
\hline 47.2 & 4 & 2 & 11.0 & 4.2 & 29.5 & 66.3 & Silty Clay \\
\hline 47.2 & 4 & 3 & 11.0 & 3.2 & 30.3 & 66.4 & Silty Clay \\
\hline 47.2 & 4 & 4 & 13.5 & 7.2 & 28.8 & 64.0 & Silty Clay \\
\hline 47.2 & 4 & 5 & 11.0 & 1.4 & 26.4 & 72.1 & Silty Clay \\
\hline 47.2 & 4 & 6 & 11.0 & 1.6 & 21.8 & 76.7 & Clay \\
\hline 47.2 & 5 & 1 & 11.0 & 1.2 & 27.6 & 71.1 & Silty Clay \\
\hline 47.2 & 5 & 2 & 1.0 & 0.9 & 22.9 & 76.1 & Clay \\
\hline 47.2 & 5 & 3 & 0.0 & 1.6 & 21.2 & 77.2 & Clay \\
\hline 47.2 & 5 & 4 & 0.5 & 5.3 & 34.2 & 60.5 & Silty Clay \\
\hline 47.2 & 5 & 5 & 0.5 & 3.3 & 34.9 & 61.8 & Silty Clay \\
\hline 47.2 & 5 & 6 & 1.0 & 3.0 & 32.4 & 64.6 & Silty Clay \\
\hline 47.2 & 6 & 2 & 0.0 & 2.9 & 31.2 & 65.9 & Silty Clay \\
\hline 47.2 & 6 & 3 & 12.0 & 2.6 & 30.4 & 67.0 & Silty Clay \\
\hline 47.2 & 6 & 3 & 122.0 & 1.9 & 31.8 & 66.3 & Silty Clay \\
\hline 47.2 & 7 & 1 & 11.0 & 0.6 & 28.3 & 71.1 & Silty Clay \\
\hline 47.2 & 7 & 1 & 77.0 & 2.7 & 35.6 & 61.7 & Silty Clay \\
\hline 47.2 & 7 & 2 & 71.0 & 2.7 & 60.4 & 36.9 & Clayey Silt \\
\hline 47.2 & 7 & 3 & 7.0 & 1.6 & 70.2 & 28.2 & Clayey Silt \\
\hline 47.2 & 7 & 4 & 11.0 & 1.0 & 68.8 & 30.2 & Clayey Silt \\
\hline 47.2 & 7 & 5 & 22.0 & 1.6 & 72.1 & 26.3 & Clayey Silt \\
\hline 47.2 & 7 & 6 & 119.5 & 2.9 & 66.7 & 30.4 & Clayey Silt \\
\hline 47.2 & 8 & 1 & 21.5 & 5.4 & 55.9 & 38.7 & Clayey Silt \\
\hline 47.2 & 8 & 2 & 11.0 & 6.9 & 56.6 & 36.5 & Clayey Silt \\
\hline 47.2 & 8 & 3 & 12.0 & 8.3 & 51.7 & 40.0 & Clayey Silt \\
\hline 47.2 & 8 & 4 & 11.0 & 10.6 & 50.1 & 39.3 & Clayey Silt \\
\hline 47.2 & 8 & 5 & 11.0 & 7.4 & 53.8 & 38.8 & Clayey Silt \\
\hline 47.2 & 8 & 6 & 11.0 & 2.0 & 48.4 & 49.6 & Silty Clay \\
\hline 47.2 & 9 & 1 & 13.0 & 4.4 & 43.5 & 52.1 & Silty Clay \\
\hline 47.2 & 9 & 2 & 11.0 & 0.2 & 38.8 & 61.0 & Silty Clay \\
\hline 47.2 & 9 & 3 & 11.0 & 0.5 & 37.3 & 62.2 & Silty Clay \\
\hline 47.2 & 9 & 4 & 0.0 & 0.3 & 37.9 & 61.8 & Silty Clay \\
\hline
\end{tabular}


TABLE 1 - Continued

\begin{tabular}{|c|c|c|c|c|c|c|c|}
\hline Hole & Core & Section & $\begin{array}{l}\text { Sampled } \\
\text { at (m) }\end{array}$ & $\begin{array}{l}\text { Sand } \\
(\%)\end{array}$ & $\begin{array}{l}\text { Silt } \\
(\%)\end{array}$ & $\begin{array}{c}\text { Clay } \\
(\%)\end{array}$ & Classification \\
\hline 47.2 & 9 & 5 & 11.0 & 0.0 & 39.0 & 60.9 & Silty Clay \\
\hline 47.2 & 9 & 6 & 0.0 & 1.0 & 35.3 & 63.7 & Silty Clay \\
\hline 47.2 & 10 & 1 & 11.0 & 1.5 & 40.5 & 58.0 & Silty Clay \\
\hline 47.2 & 10 & 2 & 11.0 & 1.0 & 38.2 & 60.7 & Silty Clay \\
\hline 47.2 & 10 & 3 & 11.0 & 0.8 & 38.4 & 60.8 & Silty Clay \\
\hline 47.2 & 10 & 5 & 11.0 & 0.6 & 30.8 & 68.6 & Silty Clay \\
\hline 47.2 & 10 & 6 & 10.0 & 0.0 & 35.4 & 64.6 & Silty Clay \\
\hline 47.2 & 11 & 1 & 145.0 & 0.8 & 47.3 & 51.9 & Silty Clay \\
\hline 47.2 & 11 & 2 & 145.0 & 0.7 & 47.4 & 51.8 & Silty Clay \\
\hline 47.2 & 11 & 3 & 145.0 & 0.8 & 42.4 & 56.8 & Silty Clay \\
\hline 47.2 & 11 & 4 & 145.0 & 0.1 & 37.9 & 62.0 & Silty Clay \\
\hline 47.2 & 11 & 5 & 145.0 & 0.1 & 32.2 & 67.7 & Silty Clay \\
\hline 47.2 & 11 & 6 & 145.0 & 0.3 & 27.6 & 72.2 & Silty Clay \\
\hline 47.2 & 12 & 1 & 145.0 & 0.4 & 27.9 & 71.7 & Silty Clay \\
\hline 47.2 & 12 & 2 & 12.0 & 0.3 & 30.6 & 69.1 & Silty Clay \\
\hline 47.2 & 12 & 3 & 145.0 & 0.1 & 28.5 & 71.3 & Silty Clay \\
\hline 47.2 & 13 & 1 & 145.0 & 2.3 & 29.6 & 68.1 & Silty Clay \\
\hline 47.2 & 13 & 2 & 145.0 & 2.0 & 35.5 & 62.5 & Silty Clay \\
\hline 47.2 & 13 & 3 & 10.0 & 1.5 & 37.3 & 61.2 & Silty Clay \\
\hline 47.2 & 13 & 4 & 145.0 & 1.8 & 27.5 & 70.7 & Silty Clay \\
\hline 47.2 & 13 & 5 & 145.0 & 0.4 & 27.6 & 71.9 & Silty Clay \\
\hline 47.2 & 13 & 6 & 145.0 & 5.3 & 30.1 & 64.7 & Silty Clay \\
\hline 47.2 & 14 & 1 & 145.0 & 6.4 & 36.9 & 56.7 & Silty Clay \\
\hline 47.2 & 14 & 2 & 145.0 & 7.2 & 37.2 & 55.6 & Silty Clay \\
\hline 47.2 & 14 & 3 & 145.0 & 4.0 & 31.0 & 64.9 & Silty Clay \\
\hline 47.2 & 14 & 4 & 145.0 & 5.8 & 33.3 & 60.8 & Silty Clay \\
\hline 47.2 & 14 & 5 & 145.0 & 6.5 & 24.5 & 69.0 & Silty Clay \\
\hline 47.2 & 14 & 6 & 145.0 & 6.9 & 40.2 & 53.0 & Silty Clay \\
\hline 48.1 & 1 & 1 & 15.0 & 2.5 & 36.1 & 61.3 & Silty Clay \\
\hline 48.2 & 1 & 1 & 16.0 & 5.0 & 28.8 & 66.2 & Silty Clay \\
\hline 48.2 & 1 & 2 & 27.0 & 2.1 & 24.5 & 73.4 & Silty Clay \\
\hline 48.2 & 1 & 3 & 10.0 & 3.3 & 27.2 & 69.5 & Silty Clay \\
\hline 48.2 & 1 & 4 & 10.0 & 3.5 & 27.0 & 69.6 & Silty Clay \\
\hline 48.2 & 1 & 5 & 1.0 & 3.2 & 26.6 & 70.2 & Silty Clay \\
\hline 48.2 & 1 & 6 & 5.0 & 2.2 & 34.5 & 63.3 & Silty Clay \\
\hline 48.2 & 2 & 1 & 145.0 & 12.5 & 34.2 & 53.2 & Silty Clay \\
\hline 48.2 & 2 & 2 & 11.0 & 10.3 & 34.6 & 55.0 & Silty Clay \\
\hline 48.2 & 2 & 3 & 145.0 & 12.5 & 30.8 & 56.7 & Silty Clay \\
\hline 48.2 & 2 & 4 & 12.0 & 18.5 & 33.9 & 47.6 & Silty Clay \\
\hline 48.2 & 2 & 5 & 10.0 & 10.8 & 29.8 & 59.4 & Silty Clay \\
\hline 48.2 & 2 & 6 & 10.0 & 8.6 & 36.3 & 55.1 & Silty Clay \\
\hline 48.2 & 3 & 1 & 114.0 & 5.7 & 25.7 & 68.6 & Silty Clay \\
\hline 48.2 & 3 & 2 & 22.0 & 7.3 & 30.0 & 62.7 & Silty Clay \\
\hline 48.2 & 3 & 3 & 8.0 & 5.8 & 28.9 & 65.4 & Silty Clay \\
\hline 48.2 & 3 & 4 & 11.0 & 9.9 & 29.5 & 60.6 & Silty Clay \\
\hline 48.2 & 3 & 5 & 10.0 & 4.5 & 31.2 & 64.2 & Silty Clay \\
\hline
\end{tabular}


TABLE 1 - Continued

\begin{tabular}{|c|c|c|c|c|c|c|c|}
\hline Hole & Core & Section & $\begin{array}{l}\text { Sampled } \\
\text { at }(\mathrm{m})\end{array}$ & $\begin{array}{c}\text { Sand } \\
(\%)\end{array}$ & $\begin{array}{l}\text { Silt } \\
(\%)\end{array}$ & $\begin{array}{l}\text { Clay } \\
(\%)\end{array}$ & Classification \\
\hline 49.0 & 1 & 1 & 5.0 & 0.7 & 23.7 & 75.6 & Clay \\
\hline 49.0 & 1 & 1 & 70.0 & 6.2 & 44.1 & 49.7 & Silty Clay \\
\hline 49.0 & 1 & 2 & 10.0 & 8.8 & 58.3 & 32.9 & Clayey Silt \\
\hline 49.0 & 1 & 3 & 10.0 & 0.5 & 42.6 & 56.9 & Silty Clay \\
\hline 49.0 & 1 & 4 & 1.0 & 7.9 & 59.4 & 32.7 & Clayey Silt \\
\hline 49.0 & 1 & 5 & 1.0 & 0.1 & 23.3 & 76.7 & Clay \\
\hline 49.0 & 1 & 6 & 1.0 & 0.5 & 23.6 & 76.0 & Clay \\
\hline 49.0 & 2 & 1 & 65.0 & 0.5 & 21.7 & 77.8 & Clay \\
\hline 49.0 & 2 & 1 & 134.0 & 2.1 & 18.2 & 79.7 & Clay \\
\hline 49.1 & 1 & 1 & 71.0 & 6.8 & 53.9 & 39.3 & Clayey Silt \\
\hline 49.1 & 1 & 2 & 10.0 & 0.1 & 29.9 & 70.0 & Silty Clay \\
\hline 49.1 & 1 & 2 & 119.0 & 2.1 & 28.2 & 69.7 & Silty Clay \\
\hline 49.1 & 1 & 3 & 17.0 & 1.5 & 30.9 & 67.7 & Silty Clay \\
\hline 49.1 & 1 & 4 & 11.0 & 1.8 & 23.4 & 74.8 & Silty Clay \\
\hline 49.1 & 1 & 4 & 111.0 & 0.0 & 29.7 & 70.3 & Silty Clay \\
\hline 49.1 & 1 & 5 & 32.0 & 8.2 & 24.1 & 67.7 & Silty Clay \\
\hline 49.1 & 1 & 5 & 84.0 & 0.8 & 21.7 & 77.5 & Clay \\
\hline 49.1 & 2 & 1 & 145.0 & 0.0 & 24.6 & 75.4 & Clay \\
\hline 49.1 & 2 & 2 & 145.0 & 1.9 & 30.1 & 68.0 & Silty Clay \\
\hline 49.1 & 2 & 3 & 145.0 & 0.0 & 16.5 & 83.5 & Clay \\
\hline 50.0 & 2 & 1 & 145.0 & 7.5 & 17.5 & 75.1 & Clay \\
\hline 50.0 & 2 & 2 & 150.0 & 14.4 & 18.0 & 67.7 & Silty Clay \\
\hline 50.0 & 2 & 3 & 145.0 & 5.9 & 16.2 & 77.9 & Clay \\
\hline 50.0 & 2 & 4 & 150.0 & 14.8 & 15.8 & 69.4 & Silty Clay \\
\hline 50.0 & 2 & 6 & 150.0 & 22.9 & 16.5 & 60.5 & Sandy Clay \\
\hline 50.1 & 1 & 3 & 1.0 & 3.0 & 33.0 & 64.0 & Silty Clay \\
\hline 50.1 & 1 & 4 & 12.0 & 2.2 & 40.9 & 56.9 & Silty Clay \\
\hline 50.1 & 1 & 5 & 10.0 & 2.3 & 42.3 & 55.4 & Silty Clay \\
\hline 50.1 & 1 & 6 & 11.0 & 2.7 & 34.1 & 63.2 & Silty Clay \\
\hline 50.1 & 2 & 1 & 126.0 & 8.2 & 50.1 & 41.7 & Clayey Silt \\
\hline 50.1 & 2 & 3 & 32.0 & 6.6 & 57.0 & 36.4 & Clayey Silt \\
\hline 50.1 & 3 & 1 & 145.0 & 0.1 & 29.0 & 70.9 & Silty Clay \\
\hline 50.1 & 3 & 2 & 70.0 & 0.2 & 17.2 & 82.6 & Clay \\
\hline 50.1 & 3 & 3 & 9.5 & 0.1 & 23.7 & 76.2 & Clay \\
\hline 50.1 & 3 & 4 & 10.0 & 0.1 & 21.3 & 78.5 & Clay \\
\hline 50.1 & 3 & 5 & 0.0 & 0.0 & 0.0 & 0.0 & Clay \\
\hline 50.1 & 3 & 6 & 10.0 & 1.7 & 21.9 & 76.3 & Clay \\
\hline 50.1 & 4 & 1 & 75.0 & 1.2 & 24.6 & 74.2 & Silty Clay \\
\hline 51.0 & 1 & 1 & 2.0 & 1.8 & 10.3 & 87.9 & Clay \\
\hline 51.0 & 1 & 2 & 1.0 & 0.1 & 9.5 & 90.4 & Clay \\
\hline 51.0 & 1 & 4 & 1.0 & 0.0 & 9.8 & 90.2 & Clay \\
\hline 51.0 & 1 & 5 & 10.0 & 0.1 & 11.0 & 89.0 & Clay \\
\hline 51.1 & 1 & 1 & 10.0 & 3.4 & 31.5 & 65.1 & Silty Clay \\
\hline 51.1 & 1 & 2 & 10.0 & 0.9 & 29.5 & 69.6 & Silty Clay \\
\hline
\end{tabular}


TABLE 1 - Continued

\begin{tabular}{|c|c|c|c|c|c|c|c|}
\hline Hole & Core & Section & $\begin{array}{c}\text { Sampled } \\
\text { at (m) }\end{array}$ & $\begin{array}{l}\text { Sand } \\
(\%)\end{array}$ & $\begin{array}{l}\text { Silt } \\
(\%)\end{array}$ & $\begin{array}{l}\text { Clay } \\
(\%)\end{array}$ & Classification \\
\hline 51.1 & 1 & 2 & 90.0 & 0.6 & 28.2 & 71.2 & Silty Clay \\
\hline 51.1 & 1 & 3 & 24.0 & 0.5 & 27.2 & 72.3 & Silty Clay \\
\hline 51.1 & 1 & 3 & 114.0 & 0.6 & 34.3 & 65.1 & Silty Clay \\
\hline 51.1 & 1 & 4 & 10.0 & 0.8 & 32.7 & 66.5 & Silty Clay \\
\hline 51.1 & 1 & 5 & 10.0 & 1.3 & 44.4 & 54.2 & Silty Clay \\
\hline 51.1 & 1 & 6 & 10.0 & 0.3 & 31.4 & 68.3 & Silty Clay \\
\hline 51.1 & 1 & 6 & 110.0 & 1.7 & 40.7 & 57.6 & Silty Clay \\
\hline 51.1 & 2 & 1 & 122.0 & 0.2 & 9.0 & 90.8 & Clay \\
\hline 51.1 & $\mathrm{x}$ & 1 & 145.0 & 1.8 & 4.9 & 93.4 & Clay \\
\hline 52.0 & 1 & 1 & 22.0 & 1.4 & 38.1 & 60.5 & Silty Clay \\
\hline 52.0 & 1 & 1 & 77.0 & 15.0 & 45.4 & 39.6 & Clayey Silt \\
\hline 52.0 & 1 & 2 & 1.0 & 6.0 & 44.6 & 49.3 & Silty Clay \\
\hline 52.0 & 1 & 3 & 1.0 & 2.1 & 36.8 & 61.1 & Silty Clay \\
\hline 52.0 & 1 & 4 & 1.0 & 0.6 & 31.6 & 67.8 & Silty Clay \\
\hline 52.0 & 1 & 5 & 3.0 & 1.0 & 29.2 & 69.8 & Silty Clay \\
\hline 52.0 & 1 & 6 & 2.0 & 0.7 & 41.3 & 57.9 & Silty Clay \\
\hline 52.0 & 2 & 2 & 1.0 & 1.0 & 28.7 & 70.4 & Silty Clay \\
\hline 52.0 & 2 & 3 & 1.0 & 1.6 & 36.5 & 61.9 & Silty Clay \\
\hline 52.0 & 2 & 4 & 1.0 & 5.8 & 50.4 & 43.8 & Clayey Silt \\
\hline 52.0 & 2 & 5 & 12.0 & 0.5 & 28.0 & 71.5 & Silty Clay \\
\hline 52.0 & 2 & 6 & 145.0 & 0.8 & 34.9 & 64.4 & Silty Clay \\
\hline 52.0 & 3 & 1 & 10.0 & 0.9 & 34.4 & 64.7 & Silty Clay \\
\hline 52.0 & 3 & 2 & 13.0 & 0.7 & 19.0 & 80.2 & Clay \\
\hline 52.0 & 3 & 3 & 10.0 & 4.0 & 59.8 & 36.2 & Clayey Silt \\
\hline 52.0 & 3 & 4 & 10.0 & 0.4 & 39.9 & 59.7 & Silty Clay \\
\hline 52.0 & 3 & 5 & 1.0 & 1.0 & 15.1 & 83.9 & Clay \\
\hline 52.0 & 3 & 6 & 1.0 & 1.6 & 39.2 & 59.3 & Silty Clay \\
\hline 52.0 & 4 & 2 & 1.0 & 1.5 & 17.3 & 81.2 & Clay \\
\hline 52.0 & 4 & 3 & 1.0 & 0.7 & 23.5 & 75.8 & Clay \\
\hline 52.0 & 4 & 4 & 1.0 & 0.9 & 18.6 & 80.5 & Clay \\
\hline 52.0 & 4 & 5 & 1.0 & 0.4 & 20.6 & 79.0 & Clay \\
\hline 52.0 & 4 & 6 & 12.0 & 0.3 & 19.1 & 80.6 & Clay \\
\hline 52.0 & 4 & 6 & 125.0 & 0.3 & 13.3 & 86.4 & Clay \\
\hline 52.0 & 5 & 1 & 121.0 & 0.1 & 10.7 & 89.3 & Clay \\
\hline 52.0 & 5 & 2 & 11.0 & 0.7 & 16.2 & 83.2 & Clay \\
\hline 52.0 & 5 & 3 & 12.0 & 0.2 & 12.6 & 87.2 & Clay \\
\hline 52.0 & 5 & 4 & 12.0 & 0.0 & 12.8 & 87.2 & Clay \\
\hline 52.0 & 5 & 4 & 72.0 & 0.1 & 12.5 & 87.4 & Clay \\
\hline 52.0 & 5 & 5 & 12.0 & 0.1 & 12.7 & 87.2 & Clay \\
\hline 52.0 & 7 & 1 & 143.0 & 0.0 & 8.9 & 91.1 & Clay \\
\hline 52.0 & 7 & 2 & 32.0 & 0.3 & 7.3 & 92.4 & Clay \\
\hline 52.0 & 7 & 2 & 143.0 & 8.2 & 23.8 & 68.0 & Silty Clay \\
\hline 52.0 & 8 & 1 & 145.0 & 4.9 & 14.8 & 80.3 & Clay \\
\hline 52.0 & 8 & 2 & 145.0 & 33.0 & 19.4 & 47.6 & Sandy Clay \\
\hline 52.0 & 8 & 3 & 16.0 & 12.3 & 14.0 & 73.7 & Silty Clay \\
\hline
\end{tabular}


TABLE 1 - Continued

\begin{tabular}{|c|c|c|c|c|c|c|c|}
\hline Hole & Core & Section & $\begin{array}{l}\text { Sampled } \\
\text { at (m) }\end{array}$ & $\begin{array}{l}\text { Sand } \\
(\%)\end{array}$ & $\begin{array}{l}\text { Silt } \\
(\%)\end{array}$ & $\begin{array}{l}\text { Clay } \\
(\%)\end{array}$ & Classification \\
\hline 52.0 & 8 & 4 & 145.0 & 34.1 & 17.9 & 48.0 & Sandy Clay \\
\hline 52.0 & 8 & 5 & 145.0 & 25.0 & 17.3 & 57.7 & Sandy Clay \\
\hline 52.0 & 8 & 6 & 145.0 & 2.7 & 9.8 & 87.5 & Clay \\
\hline 53.0 & 1 & 1 & 10.0 & 8.9 & 66.9 & 24.1 & Clayey Silt \\
\hline 53.0 & 1 & 1 & 36.0 & 36.9 & 44.3 & 18.8 & Sandy Silt \\
\hline 53.0 & 1 & 2 & 119.0 & 2.8 & 76.3 & 20.9 & Silt \\
\hline 53.0 & 1 & 3 & 12.0 & 0.3 & 63.8 & 35.9 & Clayey Silt \\
\hline 53.0 & 1 & 3 & 123.0 & 0.8 & 69.3 & 29.9 & Clayey Silt \\
\hline 53.0 & 2 & 1 & 150.0 & 0.5 & 73.8 & 25.7 & Clayey Silt \\
\hline 53.0 & 3 & 1 & 6.0 & 1.3 & 64.3 & 34.3 & Clayey Silt \\
\hline 53.0 & 4 & 1 & 6.0 & 0.3 & 9.9 & 89.8 & Clay \\
\hline 53.0 & 4 & 1 & 10.0 & 10.9 & 48.6 & 40.5 & Clayey Silt \\
\hline 53.0 & 4 & 1 & 14.0 & 3.7 & 25.5 & 70.8 & Silty Clay \\
\hline 53.0 & 4 & 1 & 87.0 & 1.0 & 9.0 & 90.0 & Clay \\
\hline 53.0 & 4 & 2 & 6.0 & 1.8 & 11.2 & 87.0 & Clay \\
\hline 53.0 & 6 & 2 & 58.0 & 62.8 & 22.3 & 14.9 & Silty Sand \\
\hline 53.0 & 6 & 2 & 60.0 & 18.3 & 32.2 & 49.5 & Silty Clay \\
\hline 53.0 & 7 & 1 & 150.0 & 50.3 & 23.4 & 26.3 & Sand-Silt-Clay \\
\hline 53.1 & 1 & 1 & 1.0 & 0.4 & 36.9 & 62.7 & Silty Clay \\
\hline 53.1 & 1 & 2 & 1.0 & 0.0 & 26.8 & 73.1 & Silty Clay \\
\hline 53.1 & 1 & 3 & 12.0 & 1.4 & 44.5 & 54.1 & Silty Clay \\
\hline 53.1 & 1 & 4 & 11.0 & 1.1 & 34.7 & 64.2 & Silty Clay \\
\hline 53.1 & 1 & 5 & 11.0 & 1.2 & 38.1 & 60.7 & Silty Clay \\
\hline 53.1 & 1 & 6 & 21.0 & 0.1 & 24.3 & 75.6 & Clay \\
\hline 53.1 & 2 & 1 & 145.0 & 50.6 & 38.0 & 11.4 & Silty Sand \\
\hline 53.1 & 2 & 2 & 145.0 & 65.3 & 25.8 & 8.9 & Silty Sand \\
\hline 53.1 & 2 & 3 & 10.0 & 76.7 & 18.2 & 5.1 & Sand \\
\hline 53.1 & 2 & 3 & 93.0 & 12.7 & 64.9 & 22.3 & Clayey Silt \\
\hline 53.1 & 2 & 4 & 1.0 & 8.4 & 74.1 & 17.5 & Clayey Silt \\
\hline 53.1 & 2 & 5 & 4.0 & 14.3 & 68.7 & 17.0 & Clayey Silt \\
\hline 53.1 & 2 & 6 & 1.0 & 12.3 & 66.5 & 21.1 & Clayey Silt \\
\hline 53.1 & 3 & 1 & 150.0 & 27.4 & 60.5 & 12.1 & Sandy Silt \\
\hline 53.1 & 3 & 2 & 10.0 & 33.9 & 51.4 & 14.7 & Sandy Silt \\
\hline 53.1 & 3 & 3 & 17.0 & 10.9 & 70.4 & 18.6 & Clayey Silt \\
\hline 53.1 & 3 & 4 & 20.0 & 14.0 & 68.3 & 17.7 & Clayey Silt \\
\hline 53.1 & 3 & 5 & 17.0 & 6.0 & 73.9 & 20.1 & Clayey Silt \\
\hline 53.2 & 1 & 1 & 15.0 & 0.3 & 35.9 & 63.8 & Silty Clay \\
\hline 53.2 & 1 & 2 & 10.0 & 5.8 & 67.8 & 26.4 & Clayey Silt \\
\hline 53.2 & 1 & 3 & 16.0 & 25.6 & 56.5 & 17.9 & Sandy Silt \\
\hline 53.2 & 1 & 4 & 1.0 & 25.5 & 58.4 & 16.1 & Sandy Silt \\
\hline 53.2 & 1 & 5 & 1.0 & 9.7 & 67.5 & 22.8 & Clayey Silt \\
\hline 53.2 & 1 & 5 & 4.0 & 15.4 & 61.5 & 23.1 & Clayey Silt \\
\hline 53.2 & 1 & 6 & 1.0 & 15.8 & 67.4 & 16.8 & Clayey Silt \\
\hline
\end{tabular}


TABLE 1 - Continued

\begin{tabular}{|c|c|c|c|c|c|c|c|}
\hline Hole & Core & Section & $\begin{array}{l}\text { Sampled } \\
\text { at (m) }\end{array}$ & $\begin{array}{l}\text { Sand } \\
(\%)\end{array}$ & $\begin{array}{l}\text { Silt } \\
(\%)\end{array}$ & $\begin{array}{l}\text { Clay } \\
(\%)\end{array}$ & Classification \\
\hline 54.0 & 1 & 1 & 1.0 & 53.7 & 32.9 & 13.3 & Silty Sand \\
\hline 54.0 & 1 & 1 & 72.0 & 2.9 & 94.9 & 2.2 & Silt \\
\hline 54.0 & 1 & 2 & 1.0 & 7.3 & 79.6 & 13.1 & Silt \\
\hline 54.0 & 1 & 3 & 48.0 & 0.6 & 88.3 & 11.2 & Silt \\
\hline 54.0 & 2 & 1 & 11.0 & 68.5 & 25.8 & 5.6 & Silty Sand \\
\hline 54.0 & 2 & 1 & 78.0 & 0.0 & 78.0 & 22.0 & Silt \\
\hline 54.0 & 2 & 2 & 6.0 & 0.1 & 70.2 & 29.7 & Clayey Silt \\
\hline 54.0 & 2 & 3 & 8.0 & 1.0 & 88.8 & 10.2 & Silt \\
\hline 54.0 & 2 & 3 & 14.0 & 1.5 & 79.3 & 19.2 & Silt \\
\hline 54.0 & 2 & 4 & 10.0 & 0.7 & 78.2 & 21.2 & Silt \\
\hline 54.0 & 3 & 1 & 140.0 & 1.9 & 66.9 & 31.2 & Clayey Silt \\
\hline 54.0 & 4 & 2 & 10.0 & 1.5 & 78.0 & 20.5 & Silt \\
\hline 54.0 & 6 & 1 & 145.0 & 46.1 & 44.5 & 9.3 & Silty Sand \\
\hline 54.0 & 6 & 2 & 10.0 & 36.0 & 55.9 & 8.1 & Sandy Silt \\
\hline 54.0 & 6 & 3 & 17.0 & 0.4 & 73.4 & 26.2 & Clayey Silt \\
\hline 54.0 & 6 & 4 & 9.0 & 13.8 & 72.6 & 13.6 & Sandy Silt \\
\hline 54.0 & 6 & 5 & 11.0 & 0.0 & 76.2 & 23.8 & Silt \\
\hline 54.0 & 7 & 1 & 132.0 & 34.6 & 54.8 & 10.6 & Sandy Silt \\
\hline 54.0 & 7 & 2 & 28.0 & 0.1 & 83.4 & 16.5 & Silt \\
\hline 54.0 & 7 & 3 & 10.0 & 1.0 & 85.2 & 13.8 & Silt \\
\hline 54.0 & 7 & 3 & 118.0 & 0.0 & 83.6 & 16.3 & Silt \\
\hline 55.0 & 1 & 1 & 1.0 & 55.4 & 19.1 & 25.6 & Clayey Sand \\
\hline 55.0 & 1 & 2 & 1.0 & 60.8 & 16.7 & 22.5 & Clayey Sand \\
\hline 55.0 & 1 & 3 & 1.0 & 44.2 & 16.5 & 39.3 & Clayey Sand \\
\hline 55.0 & 1 & 4 & 1.0 & 30.9 & 21.9 & 47.2 & Sand-Silt-Clay \\
\hline 55.0 & 1 & 5 & 1.0 & 42.3 & 18.2 & 39.5 & Clayey Sand \\
\hline 55.0 & 1 & 6 & 1.0 & 34.0 & 25.2 & 40.8 & Sand-Silt-Clay \\
\hline 55.0 & 2 & 1 & 1.0 & 36.6 & 22.5 & 40.9 & Sand-Sitl-Clay \\
\hline 55.0 & 2 & 2 & 1.0 & 34.2 & 26.2 & 39.6 & Sand-Silt-Clay \\
\hline 55.0 & 2 & 3 & 10.0 & 16.9 & 22.8 & 60.2 & Silty Clay \\
\hline 55.0 & 2 & 4 & 9.0 & 16.3 & 23.9 & 59.8 & Silty Clay \\
\hline 55.0 & 2 & 5 & 1.0 & 24.1 & 24.3 & 51.7 & Sand-Silt-Clay \\
\hline 55.0 & 2 & 6 & 26.0 & 15.5 & 27.1 & 57.4 & Silty Clay \\
\hline 55.0 & 3 & 1 & 10.0 & 20.1 & 25.5 & 54.4 & Sand-Silt-Clay \\
\hline 55.0 & 3 & 3 & 8.0 & 12.7 & 29.2 & 58.2 & Silty Clay \\
\hline 55.0 & 3 & 5 & 9.0 & 21.3 & 29.5 & 49.2 & Sand-Silt-Clay \\
\hline 55.0 & 4 & 1 & 8.0 & 7.5 & 42.7 & 49.8 & Silty Clay \\
\hline 55.0 & 4 & 2 & 145.0 & 7.3 & 42.6 & 50.1 & Silty Clay \\
\hline 55.0 & 4 & 3 & 1.0 & 6.7 & 46.8 & 46.5 & Clayey Silt \\
\hline 55.0 & 4 & 4 & 145.0 & 6.9 & 52.5 & 40.6 & Clayey Silt \\
\hline 55.0 & 4 & 5 & 1.0 & 6.5 & 51.3 & 42.2 & Clayey Silt \\
\hline 55.0 & 4 & 6 & 145.0 & 1.9 & 52.1 & 46.0 & Clayey Silt \\
\hline 55.0 & 5 & 1 & 1.0 & 3.0 & 55.6 & 41.4 & Clayey Silt \\
\hline 55.0 & 5 & 3 & 2.0 & 1.1 & 53.0 & 45.9 & Clayey Silt \\
\hline 55.0 & 5 & 5 & 1.0 & 1.4 & 56.4 & 42.2 & Clayey Silt \\
\hline
\end{tabular}


TABLE 1 - Continued

\begin{tabular}{|c|c|c|c|c|c|c|c|}
\hline Hole & Core & Section & $\begin{array}{l}\text { Sampled } \\
\text { at (m) }\end{array}$ & $\begin{array}{l}\text { Sand } \\
(\%)\end{array}$ & $\begin{array}{l}\text { Silt } \\
(\%)\end{array}$ & $\begin{array}{l}\text { Clay } \\
(\%)\end{array}$ & Classification \\
\hline 55.0 & 6 & 1 & 1.0 & 2.1 & 52.3 & 45.6 & Clayey Silt \\
\hline 55.0 & 6 & 3 & 11.0 & 0.5 & 56.4 & 43.1 & Clayey Silt \\
\hline 55.0 & 6 & 5 & 10.0 & 1.4 & 54.2 & 44.5 & Clayey Silt \\
\hline 55.0 & 7 & 1 & 13.0 & 4.7 & 55.2 & 40.1 & Clayey Silt \\
\hline 55.0 & 7 & 3 & 12.0 & 4.7 & 57.1 & 38.1 & Clayey Silt \\
\hline 55.0 & 7 & 5 & 12.0 & 5.2 & 56.6 & 38.2 & Clayey Silt \\
\hline 55.0 & 8 & 1 & 9.0 & 2.2 & 57.7 & 40.1 & Clayey Silt \\
\hline 55.0 & 8 & 3 & 10.0 & 2.0 & 59.1 & 39.0 & Clayey Silt \\
\hline 55.0 & 8 & 5 & 12.0 & 8.9 & 56.4 & 34.7 & Clayey Silt \\
\hline 55.0 & 9 & 1 & 0 & 3.4 & 60.8 & 35.8 & Clayey Silt \\
\hline 55.0 & 10 & 2 & 8.0 & 4.7 & 49.9 & 45.4 & Clayey Silt \\
\hline 55.0 & 10 & 3 & 21.0 & 4.2 & 45.1 & 50.7 & Silty Clay \\
\hline 55.0 & 10 & 4 & 2.0 & 4.7 & 49.3 & 46.0 & Clayey Silt \\
\hline 55.0 & 11 & 1 & 40.0 & 13.1 & 55.9 & 31.0 & Clayey Silt \\
\hline 55.0 & 11 & 3 & 11.0 & 2.0 & 64.8 & 33.2 & Clayey Silt \\
\hline 55.0 & 11 & 5 & 1.0 & 3.2 & 63.7 & 33.1 & Clayey Silt \\
\hline 55.0 & 12 & 1 & 7.0 & 4.2 & 55.9 & 39.8 & Clayey Silt \\
\hline 55.0 & 12 & 2 & 10.0 & 5.0 & 59.6 & 35.4 & Clayey Silt \\
\hline 55.0 & 12 & 5 & 1.0 & 0.6 & 61.5 & 37.9 & Clayey Silt \\
\hline 55.0 & 13 & 1 & 4.0 & 5.2 & 57.7 & 37.1 & Clayey Silt \\
\hline 55.0 & 13 & 5 & 1.0 & 2.0 & 53.2 & 44.8 & Clayey Silt \\
\hline 55.0 & 14 & 1 & 145.0 & 0.5 & 58.2 & 41.2 & Clayey Silt \\
\hline 55.0 & 14 & 2 & 145.0 & 1.2 & 60.0 & 38.8 & Clayey Silt \\
\hline 55.0 & 14 & 3 & 145.0 & 0.8 & 59.8 & 39.4 & Clayey Silt \\
\hline 55.0 & 14 & 4 & 145.0 & 0.6 & 60.5 & 38.9 & Clayey Silt \\
\hline 55.0 & 14 & 5 & 145.0 & 0.5 & 57.8 & 41.7 & Clayey Silt \\
\hline 55.0 & 14 & 6 & 145.0 & 0.4 & 60.8 & 38.8 & Clayey Silt \\
\hline 56.2 & 1 & 2 & 145.0 & 9.7 & 27.7 & 62.6 & Silty Clay \\
\hline 56.2 & 1 & 4 & 20.0 & 9.6 & 33.6 & 56.9 & Silty Clay \\
\hline 56.2 & 1 & 6 & 1.0 & 2.0 & 40.0 & 58.0 & Silty Clay \\
\hline 56.2 & 2 & 2 & 90.0 & 7.4 & 43.3 & 49.3 & Silty Clay \\
\hline 56.2 & 2 & 4 & 30.0 & 16.2 & 36.3 & 47.4 & Silty Clay \\
\hline 56.2 & 2 & 6 & 10.0 & 20.0 & 35.0 & 44.9 & Sand-Silt-Clay \\
\hline 56.2 & 3 & 6 & 145.0 & 11.9 & 29.8 & 58.4 & Silty Clay \\
\hline 56.2 & 4 & 4 & 7.0 & 12.8 & 59.9 & 27.3 & Clayey Silt \\
\hline 56.2 & 4 & 6 & 7.0 & 11.5 & 49.7 & 38.8 & Clayey Silt \\
\hline 56.2 & 5 & 2 & 8.0 & 9.2 & 53.2 & 37.6 & Clayey Silt \\
\hline 56.2 & 5 & 6 & 1.0 & 14.7 & 46.6 & 38.7 & Clayey Silt \\
\hline 56.2 & 6 & 2 & 1.0 & 16.1 & 53.9 & 30.1 & Clayey Silt \\
\hline 56.2 & 6 & 4 & 1.0 & 13.1 & 51.3 & 35.6 & Clayey Silt \\
\hline 56.2 & 7 & 2 & 38.0 & 13.6 & 48.0 & 38.5 & Clayey Silt \\
\hline 56.2 & 7 & 2 & 108.0 & 22.8 & 55.9 & 21.3 & Sand-Silt-Clay \\
\hline 56.2 & 7 & 4 & 34.0 & 21.6 & 46.9 & 31.4 & Sand-Silt-Clay \\
\hline 56.2 & 7 & 6 & 1.0 & 9.2 & 55.7 & 35.1 & Clayey Silt \\
\hline 56.2 & 8 & 4 & 1.0 & 17.8 & 51.9 & 30.3 & Clayey Silt \\
\hline
\end{tabular}


TABLE 1 - Continued

\begin{tabular}{|c|c|c|c|c|c|c|c|}
\hline Hole & Core & Section & $\begin{array}{l}\text { Sampled } \\
\text { at (m) }\end{array}$ & $\begin{array}{l}\text { Sand } \\
(\%)\end{array}$ & $\begin{array}{l}\text { Silt } \\
(\%)\end{array}$ & $\begin{array}{l}\text { Clay } \\
(\%)\end{array}$ & Classification \\
\hline 56.2 & 9 & 2 & 1.0 & 21.7 & 46.2 & 32.2 & Sand-Silt-Clay \\
\hline 56.2 & 9 & 4 & 12.0 & 32.0 & 45.9 & 22.1 & Sand-Silt-Clay \\
\hline 56.2 & 9 & 6 & 2.0 & 22.7 & 52.1 & 25.3 & Sand-Silt-Clay \\
\hline 56.2 & 9 & 6 & 92.0 & 21.5 & 54.0 & 24.5 & Sand-Silt-Clay \\
\hline 56.2 & 10 & 2 & 4.0 & 21.9 & 48.6 & 29.5 & Sand-Silt-Clay \\
\hline 56.2 & 10 & 3 & 1.0 & 28.8 & 41.2 & 30.0 & Sand-Silt-Clay \\
\hline 57.0 & 1 & 1 & 5.0 & 7.6 & 44.3 & 48.1 & Silty Clay \\
\hline 57.1 & 1 & 1 & 8.0 & 6.7 & 34.1 & 59.1 & Silty Clay \\
\hline 57.1 & 1 & 2 & 0.0 & 11.2 & 45.7 & 43.0 & Clayey Silt \\
\hline 57.1 & 1 & 2 & 7.0 & 3.6 & 39.2 & 57.2 & Silty Clay \\
\hline 57.1 & 1 & 3 & 1.0 & 4.0 & 36.2 & 59.7 & Silty Clay \\
\hline 57.1 & 1 & 4 & 1.0 & 6.6 & 44.1 & 49.3 & Silty Clay \\
\hline 57.1 & 1 & 6 & 5.0 & 6.3 & 35.0 & 58.8 & Silty Clay \\
\hline 57.1 & 2 & 1 & 36.0 & 11.0 & 45.5 & 43.5 & Clayey Silt \\
\hline 57.1 & 3 & 1 & 15.0 & 14.9 & 45.6 & 39.5 & Clayey Silt \\
\hline 57.1 & 3 & 2 & 27.0 & 26.6 & 58.3 & 15.1 & Sandy Silt \\
\hline 57.1 & 3 & 2 & 104.0 & 7.9 & 46.1 & 46.0 & Clayey Silt \\
\hline 57.1 & 4 & 1 & 8.0 & 7.7 & 50.3 & 41.9 & Clayey Silt \\
\hline 57.1 & 4 & 2 & 27.0 & 15.7 & 50.3 & 34.0 & Clayey Silt \\
\hline 57.1 & 4 & 3 & 11.0 & 11.6 & 47.5 & 40.9 & Clayey Silt \\
\hline 57.1 & 4 & 4 & 145.0 & 8.6 & 50.0 & 41.5 & Clayey Silt \\
\hline 57.1 & 4 & 5 & 145.0 & 14.8 & 45.8 & 39.4 & Clayey Silt \\
\hline 57.2 & 1 & 1 & 16.0 & 7.3 & 22.8 & 69.9 & Silty Clay \\
\hline 58.1 & 1 & 1 & 133.0 & 1.9 & 24.4 & 73.6 & Silty Clay \\
\hline 58.1 & 1 & 2 & 19.0 & 3.4 & 32.6 & 64.0 & Silty Clay \\
\hline 58.1 & 1 & 3 & 145.0 & 85.5 & 7.1 & 7.4 & Sand \\
\hline 58.2 & 1 & 1 & 60.0 & 83.3 & 8.8 & 7.8 & Sand \\
\hline 58.2 & 1 & 1 & 102.0 & 1.3 & 52.1 & 46.7 & Clayey Silt \\
\hline 58.2 & 1 & 3 & 10.0 & 7.9 & 43.8 & 48.3 & Silty Clay \\
\hline 58.2 & 1 & 4 & 26.0 & 7.2 & 49.6 & 43.2 & Clayey Silt \\
\hline 58.2 & 1 & 4 & 130.0 & 7.9 & 82.3 & 9.8 & Silt \\
\hline 59.2 & 1 & 3 & 7.0 & 2.4 & 61.8 & 35.8 & Clayey Silt \\
\hline 59.2 & 2 & 2 & 145.0 & 7.5 & 66.8 & 25.7 & Clayey Silt \\
\hline 59.2 & 2 & 3 & 1.0 & 9.3 & 55.3 & 35.4 & Clayey Silt \\
\hline 59.2 & 2 & 4 & 1.0 & 16.9 & 31.7 & 51.5 & Silty Clay \\
\hline 59.2 & 3 & 1 & 145.0 & 6.7 & 70.4 & 22.9 & Clayey Silt \\
\hline 59.2 & 3 & 2 & 145.0 & 0.6 & 45.4 & 54.0 & Silty Clay \\
\hline 59.2 & 4 & 1 & 60.0 & 6.3 & 55.5 & 38.3 & Clayey Silt \\
\hline 59.2 & 5 & 1 & 82.0 & 0.3 & 87.7 & 12.1 & Silt \\
\hline 59.2 & 6 & 2 & 0.0 & 18.1 & 32.5 & 49.3 & Silty Clay \\
\hline 60.0 & 3 & 1 & 111.0 & 0.0 & 76.7 & 23.3 & Silt \\
\hline 60.0 & 3 & 2 & 4.0 & 0.0 & 65.2 & 34.8 & Clayey Silt \\
\hline 60.0 & 3 & 2 & 121.0 & 47.7 & 45.8 & 6.5 & Silty Sand \\
\hline 60.0 & 4 & 1 & 145.0 & 2.3 & 73.2 & 24.5 & Clayey Silt \\
\hline
\end{tabular}


TABLE 1 - Continued

\begin{tabular}{rccrrrrl}
\hline Hole & Core & Section & $\begin{array}{c}\text { Sampled } \\
\text { at }(\mathrm{m})\end{array}$ & $\begin{array}{c}\text { Sand } \\
(\%)\end{array}$ & $\begin{array}{c}\text { Silt } \\
(\%)\end{array}$ & $\begin{array}{c}\text { Clay } \\
(\%)\end{array}$ & Classification \\
\hline 60.0 & 4 & 2 & 145.0 & 6.3 & 77.4 & 16.3 & Silt \\
60.0 & 5 & 1 & 44.0 & 0.0 & 73.1 & 26.9 & Clayey Silt \\
60.0 & 5 & 2 & 20.0 & 1.3 & 78.0 & 20.7 & Silt \\
60.0 & 6 & 1 & 79.0 & 2.7 & 65.3 & 32.0 & Clayey Silt \\
60.0 & 6 & 2 & 10.0 & 0.2 & 67.8 & 32.1 & Clayey Silt \\
60.0 & 6 & 3 & 7.0 & 17.0 & 72.2 & 10.7 & Sandy Silt \\
60.0 & 6 & 6 & 10.0 & 21.1 & 45.1 & 33.8 & Sand-Silt-Clay \\
60.0 & 6 & 7 & 101.0 & 48.0 & 43.1 & 8.9 & Silty Sand \\
60.0 & 7 & 1 & 75.0 & 5.5 & 83.0 & 1.5 & Silt \\
60.0 & 7 & 2 & 2.0 & 11.2 & 70.9 & 17.9 & Clayey Silt \\
60.0 & 8 & 2 & 1.0 & 13.7 & 48.6 & 37.7 & Clayey Silt \\
60.0 & 9 & 3 & 81.0 & 60.7 & 30.7 & 8.6 & Silty Sand \\
\hline
\end{tabular}



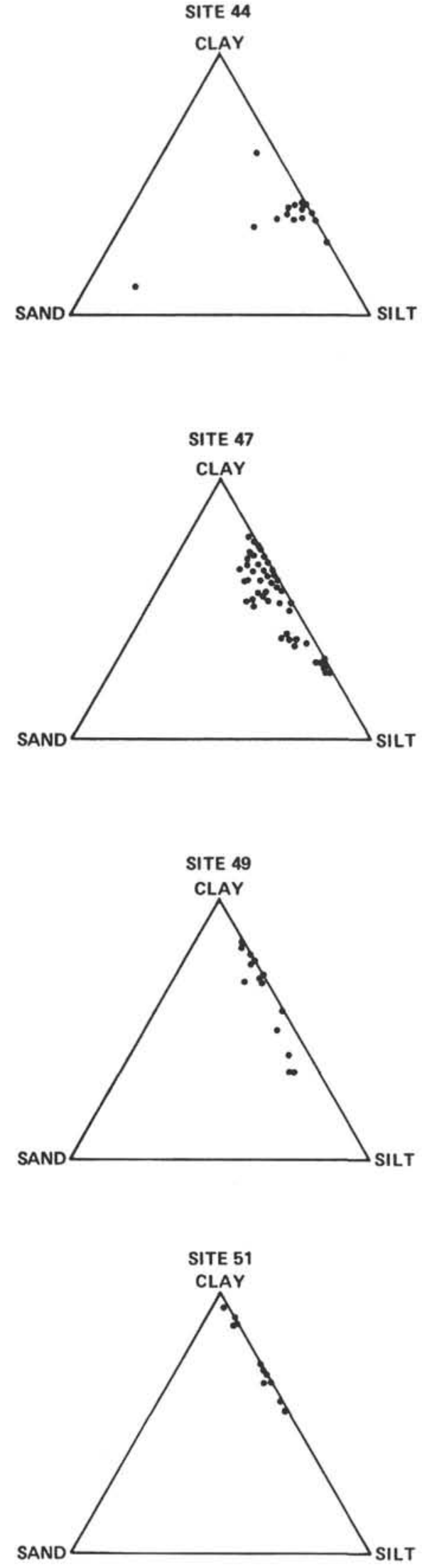
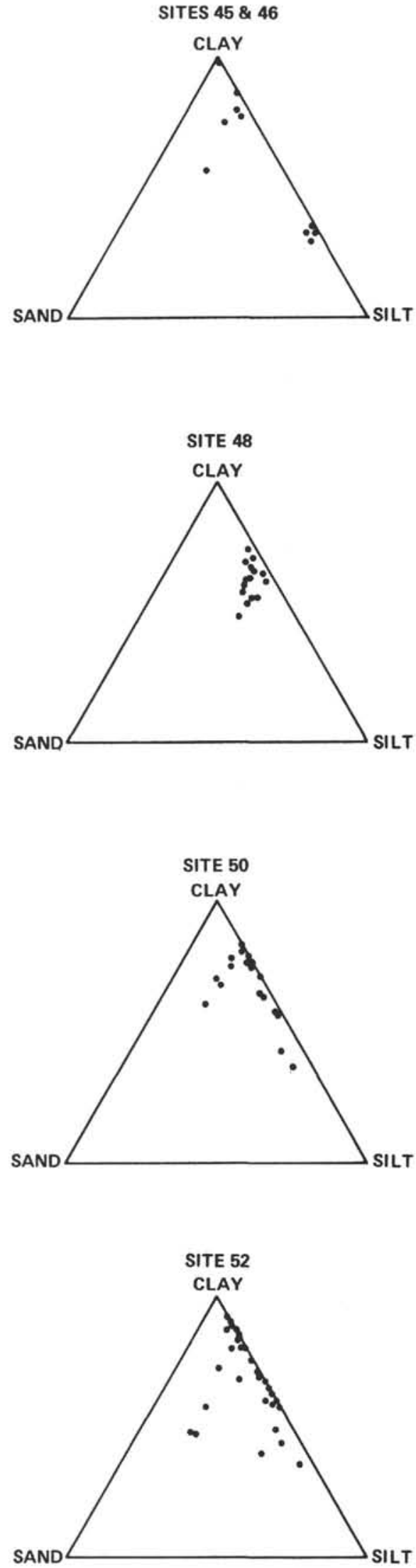

Figure 1. Triangle diagrams of grain size for Sites from Leg 6. 

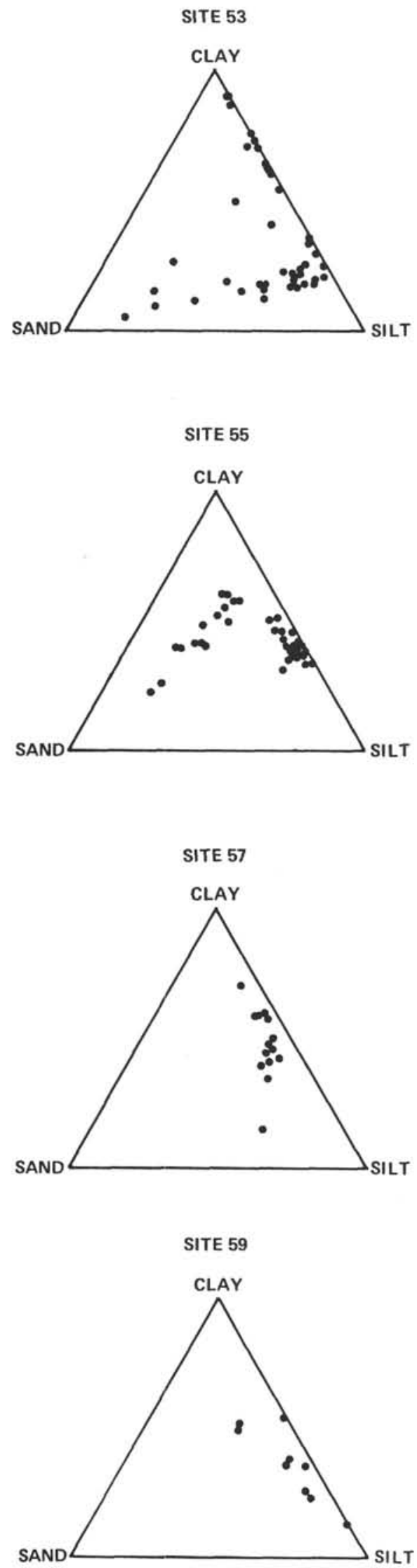
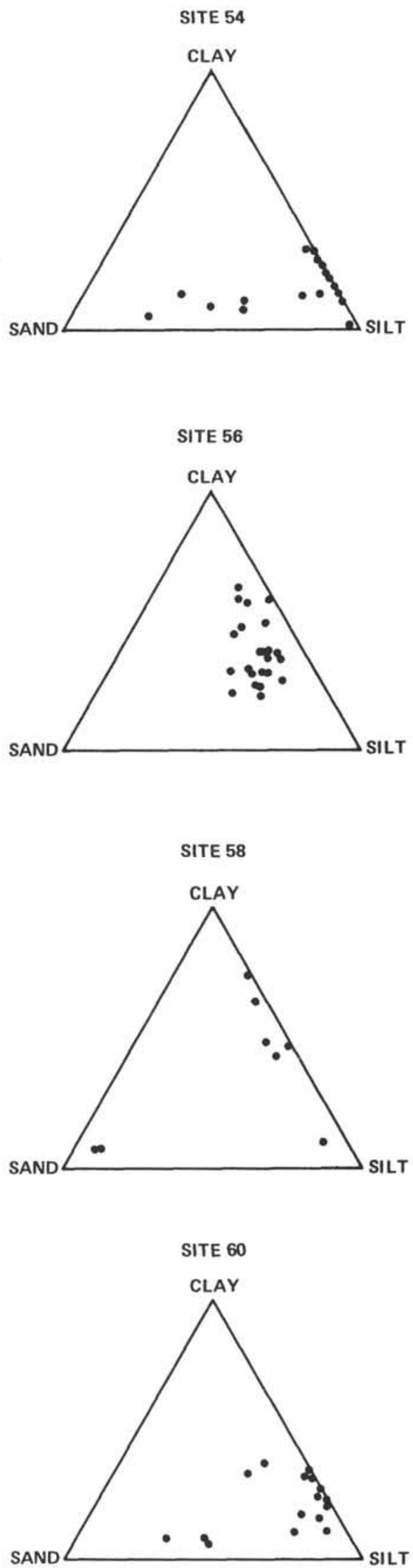

Figure 1. (Continued). 

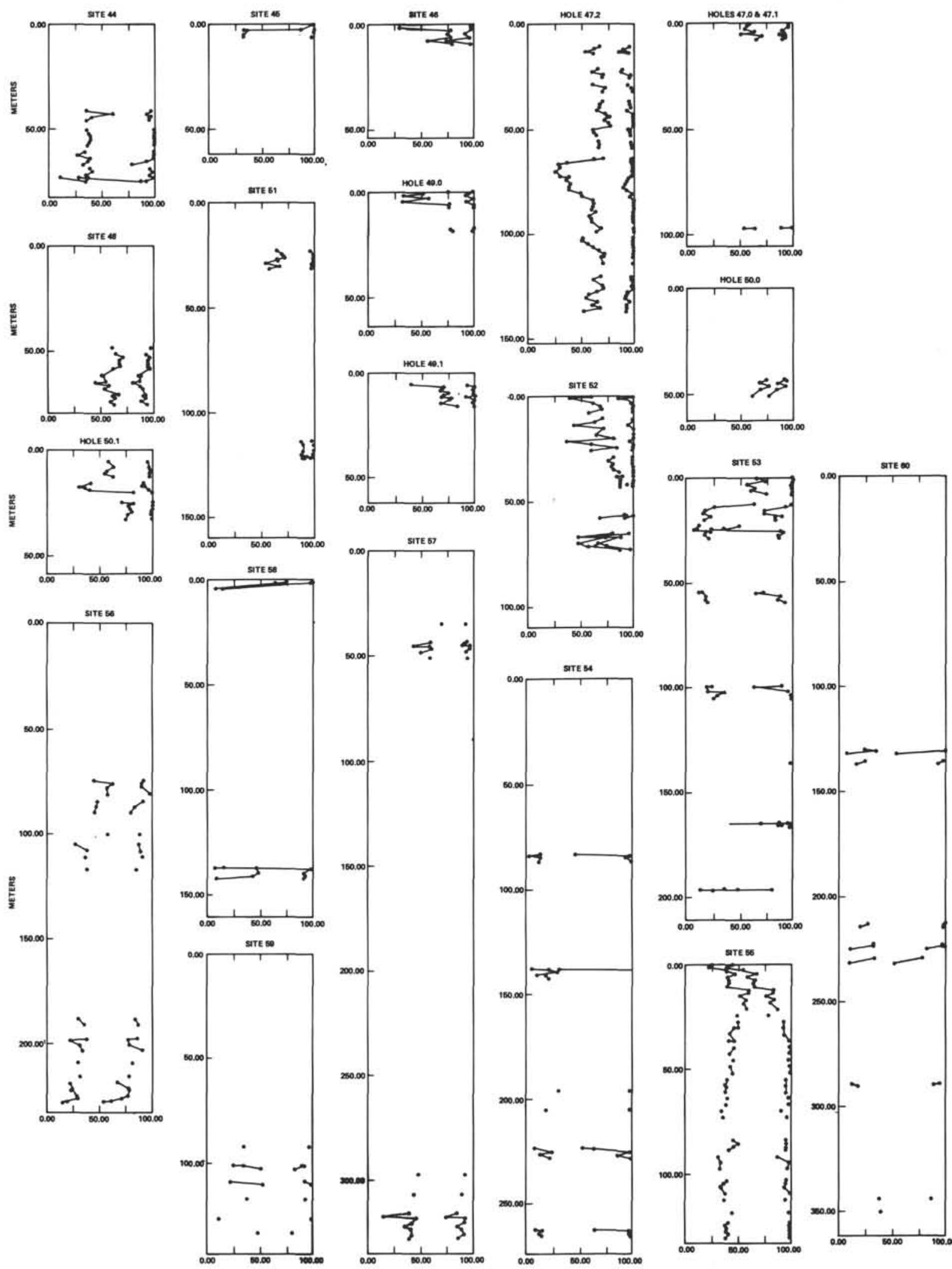

Figure 2. Down-hole plots of grain size for Leg 6 Sites. Left hand curve shows per cent clay, right hand curve per cent silt plus clay $(=100-$ per cent sand). 
Hole 53.2 mainly recovered siliceous ooze in which the silt fraction predominates, variations in sand content are related to the abundance of Radiolaria.

\section{Site 54}

One hole drilled at this site recovered volcanic ash mainly in the silt or, less commonly, clayey silt and sandy silt size range.

\section{Site 55}

One hole drilled at this site recovered a thick sequence of chalk ooze whose grain size reflects closely its calcareous fossil content. In Cores 1 and 2, foraminifera are usually more abundant than nannoplankton so the sediment mainly falls in the clayey sand or sand-siltclay size range. In Core 3 , foraminifera are less common and are slightly subordinate to nannoplankton, therefore, the sediment is mainly silty clay size.

Cores 4 through 14 are all of clayey silt size, as nannoplankton are predominant; foraminifera only occur in small amounts. The sharp break in grain size classification between Cores 1, 2 and 3 and Cores 4 through 14 is particularly interesting as there is a time gap of approximately 9 million years between Core 3 (Late Pliocene) and Core 4 (Early late Miocene).

\section{Site 56}

This hole penetrated a thick sequence of chalk ooze whose grain size analysis closely reflects its calcareous fossil content. Silty clay and clayey silt predominate in Cores 1 through 7 indicating a high nannoplankton content with smaller amounts of foraminifera (the sand content usually being less than 15 per cent). In Cores 8 through 10 , foraminifera are more abundant and the sediment has a sandy silt or sand-silt-clay size range.

\section{Site 57}

Three holes drilled at this site mainly recovered nannoplankton marl and chalk ooze in the silty clay or clayey silt size range. A few thin volcanic ash interbeds also occur and these fall in the sandy silt size (57.1-3-2, 27 centimeters).

\section{Site 58}

Siliceous and carbonate ooze in Sections 1 and 2, Core 1 of Hole 58.1 are of silty clay size, while skeletal sand (mainly shell and echinoderm fragments) occur in the lower part of Section 3. In Hole 58.2, a mixture of siliceous and carbonate ooze falls in the clayey silt or silty clay size, while pebbly mudstone from Section 1 , Core 1 , falls in the sand-size range.

\section{Site 59}

Siliceous ooze interbedded and mixed with pelagic brown clay falls in the clayey silt-and silty clay-size range. In Core 5, Section 1, a thin ash interbed contained over 80 per cent silt.

\section{Site 60}

Volcanic ash recovered at this site was predominantly in the clayey silt-and silt-size range, but in several places coarser material falls in the silty sand class.

\section{COMPOSITION OF SAND FRACTION}

\section{Introduction}

The sand fraction recovered from the grain size analysis was examined under a binocular microscope. A complete summary of the composition of the sand fraction is given in Table 2, and a detailed description by holes below. In brief, the sand fraction of chalk oozes is dominated by planktonic foraminifera. Siliceous microfossils are rare in the chalk oozes and are usually only present in Upper Oligocene and younger sediment being most common in the Caroline Ridge sites. Detrital and authigenic minerals are extremely rare and probably make up less than 1 per cent of the total sand fraction.

Siliceous oozes are dominated by siliceous fossils in their sand fraction and, like calcareous oozes, all other constituents are rare.

In pelagic brown clay, authigenic and volcanic minerals predominate in the sand fraction; siliceous fossils may occur in abundance in a few places, but are usually rare. Fish debris and, in places, manganese are common constituents of the sand fraction in brown clay, but detrital minerals are rare.

Volcanic ash has a sand fraction dominated by glass and, to a lesser extent, pumice. Biogenic components and rock fragments usually occur in small amounts. Angular fragments of chert occur in all sediment types wherever the drill penetrates these beds.

\section{Site 44}

The sand fraction here is dominated by planktonic foraminifera. Minor constituents include benthonic foraminifera, echinoid spines, ostracods, and fish debris with rare grains of manganese.

Additions to the above suite include carbonate grains in Core 3 ; colorless to pale brown chert occurs in Cores 3, 4 and 5, and bedded red chert in Core 3; lithified chalk fragments were also seen in Core 3.

\section{Site 45}

The sand fraction in Hole 45.1 is predominantly manganese with minor limonite. Many of the manganese grains have a distinctive rounded shape suggestive of a 
TABLE 2

Summary of the Composition of the Coarse Fraction, Leg 6

\begin{tabular}{|c|c|c|c|c|c|c|c|c|c|c|c|c|c|c|c|c|c|c|c|c|c|}
\hline \multicolumn{3}{|c|}{ SAMPLE NO. } & \multirow{2}{*}{ 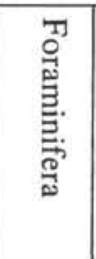 } & \multirow{2}{*}{ 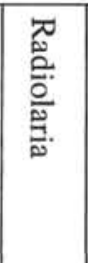 } & \multirow{2}{*}{ 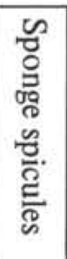 } & \multirow{2}{*}{ 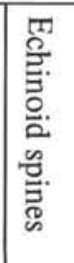 } & \multirow{2}{*}{ 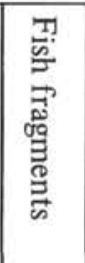 } & \multirow{2}{*}{ 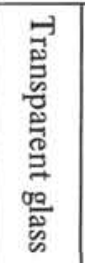 } & \multirow{2}{*}{ 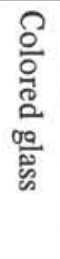 } & \multirow{2}{*}{ 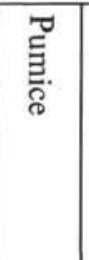 } & \multirow{2}{*}{ 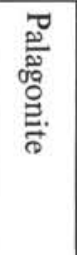 } & \multirow{2}{*}{ 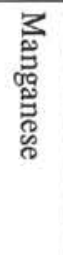 } & \multirow{2}{*}{ 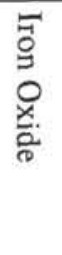 } & \multirow{2}{*}{ 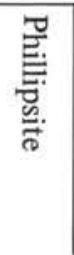 } & \multirow{2}{*}{ 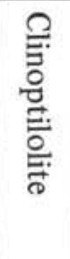 } & \multirow{2}{*}{ 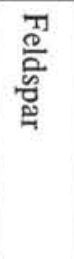 } & \multirow{2}{*}{$\begin{array}{l}\stackrel{O}{\mathrm{E}} \\
\stackrel{\mathrm{E}}{\mathrm{N}}\end{array}$} & \multirow{2}{*}{ 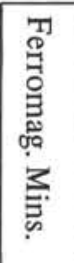 } & \multirow{2}{*}{ 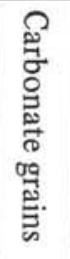 } & \multirow{2}{*}{ 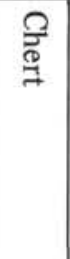 } & \multirow{2}{*}{ 옳 } \\
\hline$\frac{T}{0}$ & ஓ & $\begin{array}{l}\text { n } \\
\stackrel{0}{0} \\
\stackrel{0}{0}\end{array}$ & & & & & & & & & & & & & & & & & & & \\
\hline 44.0 & 1 & $1-4$ & D & & & $\mathrm{R}$ & $\mathrm{R}$ & & & & & & $\mathrm{R}$ & & & & & & & & Ostracod R \\
\hline 44.0 & 2 & $1-6$ & D & & & $\mathrm{R}$ & $\mathrm{R}$ & & & & & & $\mathrm{R}$ & & & & & & & & \\
\hline 44.0 & 3 & $2 \cdot 4$ & $\mathrm{D}$ & & & $\mathrm{R}$ & $\mathrm{R}$ & & & & & $R$ & $\mathrm{R}$ & & & & & & $\mathrm{R}$ & $\mathrm{R}$ & \\
\hline 44.0 & 3 & $5, \mathrm{CC}$ & $\mathrm{D}$ & & & $\mathrm{R}$ & & & & & & $R$ & $\mathrm{R}$ & & & & & & $\mathrm{C}$ & A & \\
\hline 44.0 & 4 & 0,2 & $\mathrm{D}$ & & & $\mathrm{R}$ & $\mathrm{R}$ & & & & & $\mathrm{R}$ & $\mathrm{R}$ & & & & & & & C & \\
\hline 44.0 & 4 & $3, \mathrm{CC}$ & D & & & $\mathrm{R}$ & $\mathrm{R}$ & & & & & $\mathrm{R}$ & & & & & & & & $\mathrm{R}$ & \\
\hline 44.0 & 5 & 0 & D & & & $\mathrm{R}$ & & & & & & $\mathrm{R}$ & $\mathrm{R}$ & & & & & & & $\mathrm{C}$ & \\
\hline 45.1 & 1 & $1-5$ & & & & & & $\mathrm{R}$ & & & C-A & A & $\mathrm{R}$ & & & & & & & & Siliceous grains- $\mathrm{R}$ \\
\hline 46.0 & 1 & $1-6$ & & $\mathrm{~A}$ & $\mathrm{R}$ & & $\mathrm{R}$ & & & & & $\mathrm{C}$ & & & & & & & & $\mathrm{C}$ & Lithified ash-C \\
\hline 47.0 & 1 & $1-6$ & A-D & $A(5)$ & $\mathrm{R}$ & $\mathrm{R}$ & & $\mathrm{A}(2)$ & & & & & & & & & & & $\mathrm{R}$ & & \\
\hline 47.1 & 1 & $1-2$ & $\mathrm{D}$ & $\mathrm{R}$ & $\mathrm{R}$ & $\mathrm{R}$ & $\mathrm{R}$ & & & & & $\mathrm{R}$ & & & & & & & $\mathrm{R}$ & & \\
\hline 47.2 & $1-4$ & All & D & $\mathrm{R}$ & $\mathrm{R}$ & $\mathrm{R}$ & $\mathrm{R}$ & & & & & $\mathrm{R}$ & & & & & & & $\mathrm{R}$ & & \\
\hline 47.2 & 5,6 & All & $\mathrm{A}$ & C-A & $\mathrm{R}$ & $\mathrm{R}$ & $\mathrm{R}$ & & & & & $\mathrm{R}$ & & & & & & & $\mathrm{R}$ & & \\
\hline 47.2 & 7 & 1 & $\mathrm{D}$ & & $\mathrm{R}$ & $\mathrm{R}$ & $\mathrm{R}$ & & & & & $\mathrm{R}$ & & $\mathrm{R}$ & & & & & & & \\
\hline 47.2 & 7 & $2-6$ & $\mathrm{D}$ & & & & & & & & & & & & & & $\mathrm{R}(5)$ & & & & \\
\hline 47.2 & 8 & $1-6$ & $\mathrm{D}$ & & & & & & & & & & & & & & & & & & \\
\hline 47.2 & 9 & $1-6$ & $\mathrm{D}$ & & & & R-C & & & $\mathrm{R}(5)$ & & & & & & & & & & & \\
\hline 47.2 & 10 & $1-6$ & $\mathrm{D}$ & & & & & & & & & & & & & & & & & & \\
\hline 47.2 & 11 & $1-6$ & $\mathrm{D}$ & & & & & $\mathrm{R}$ & & & & & & & & & & & & & Inoceramus- $R$ \\
\hline 47.2 & 12 & 1,2 & R-C & & & & & & & & $\mathrm{R}$ & $\mathrm{C}$ & & & & & & & & $\mathrm{A}$ & \\
\hline 47.2 & 12 & 3 & $\mathrm{D}$ & & & & & & $\mathrm{R}$ & & & & & & & & $\mathrm{R}$ & & & & \\
\hline 47.2 & 13 & $1-6$ & $\mathrm{D}$ & & & & & & & & $\mathrm{R}$ & & & & & & & & & $\mathrm{R}$ & \\
\hline 48.1 & 1 & 1 & $\mathrm{~A}$ & $\mathrm{R}$ & $\mathrm{R}$ & $\mathrm{R}$ & & & $\mathrm{R}$ & & $\mathrm{A}$ & & & & & & & & & & \\
\hline 48.2 & 1 & 1 & $\mathrm{~A}$ & & & & & & & & & & & & & & & & $\mathrm{~A}$ & & \\
\hline 48.2 & 1 & 2 & $\mathrm{~A}$ & $\mathrm{R}$ & $\mathrm{R}$ & & & & $\mathrm{R}$ & & & $\mathrm{R}$ & & & & & & & & A? & \\
\hline
\end{tabular}


TABLE 2 - Continued

\begin{tabular}{|c|c|c|c|c|c|c|c|c|c|c|c|c|c|c|c|c|c|c|c|c|c|}
\hline \multicolumn{3}{|c|}{ SAMPLE NO. } & \multirow{2}{*}{ 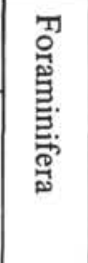 } & \multirow{2}{*}{ 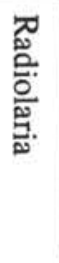 } & \multirow{2}{*}{ 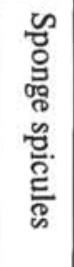 } & \multirow{2}{*}{ 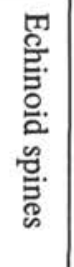 } & \multirow{2}{*}{ 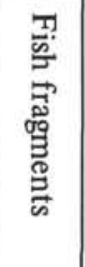 } & \multirow{2}{*}{ 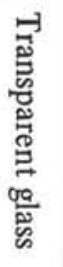 } & \multirow{2}{*}{ 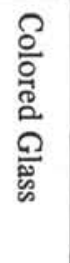 } & \multirow{2}{*}{ 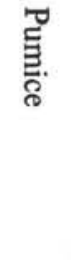 } & \multirow{2}{*}{ 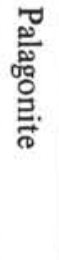 } & \multirow{2}{*}{ 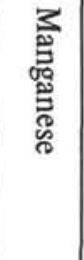 } & \multirow{2}{*}{ 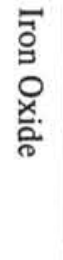 } & \multirow{2}{*}{ 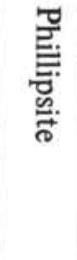 } & \multirow{2}{*}{ 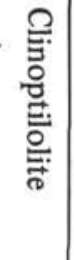 } & \multirow{2}{*}{ 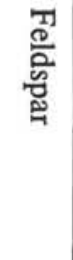 } & \multirow{2}{*}{$\begin{array}{l}\text { 을 } \\
\text { 产 }\end{array}$} & \multirow{2}{*}{ 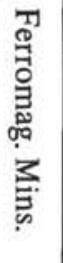 } & \multirow{2}{*}{ 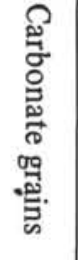 } & \multirow{2}{*}{$\stackrel{?}{\stackrel{7}{7}}$} & \multirow{2}{*}{ 옳 } \\
\hline$\frac{\text { T뭉 }}{0}$ & ஓ & 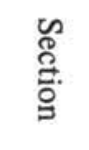 & & & & & & & & & & & & & & & & & & & \\
\hline 48.2 & 1 & $3-5$ & A & $\mathrm{R}$ & $\mathrm{R}$ & $\mathrm{R}$ & $\mathrm{R}$ & $\mathrm{C}$ & & & & & & C & & & & & $\mathrm{C}(5)$ & & Ostracod (5) \\
\hline 49.0 & 1 & $1 \cdot 6$ & & $\mathrm{R}$ & & & $\mathrm{R}$ & A & & & A & $\mathrm{A}$ & & $\mathrm{R}$ & & $\mathrm{R}$ & & & & & \\
\hline 49.0 & 2 & 1 & A & $\mathrm{R}$ & & & $\mathrm{R}$ & $\mathrm{R}$ & & & $\mathrm{R}$ & $\mathrm{A}$ & & $\mathrm{R}$ & & & & & A & & \\
\hline 49.1 & 1 & 1 & & $\mathrm{R}$ & & & & A & & & $\mathrm{A}$ & A & & & & & & & & & \\
\hline 49.1 & 1 & 2,3 & & & & & $\mathrm{C}$ & & & & & A & & A & & $\mathrm{R}$ & & & & & \\
\hline 49.1 & 1 & 4 & $\mathrm{R}$ & $\mathrm{R}$ & & & $\mathrm{R}$ & & & & A & $\mathrm{A}$ & & $\mathrm{R}$ & & & & & & & \\
\hline 50.1 & 1 & 4 & $\mathrm{C}$ & $\mathrm{R}$ & $\mathrm{R}$ & $\mathrm{R}$ & & $\mathrm{A}$ & & $\mathrm{A}$ & $\mathrm{C}$ & $\mathrm{R}$ & & & & & & & & & \\
\hline 50.1 & 1 & 5 & $\mathrm{D}$ & & $\mathrm{R}$ & $\mathrm{C}$ & & & & & $\mathrm{R}$ & & & & & & & & & & \\
\hline 50.1 & 1 & 6 & $\mathrm{C}$ & $\mathrm{R}$ & $\mathrm{R}$ & $\mathrm{R}$ & & A & & A & $\mathrm{C}$ & $\mathrm{R}$ & & & & & & & & & \\
\hline 50.1 & 2 & 1,3 & & & $\mathrm{R}$ & & $\mathrm{R}$ & $\mathrm{A}$ & & & $\mathrm{A}$ & $\mathrm{A}$ & & & & $\mathrm{R}$ & & & & & \\
\hline 50.1 & 3 & $1 \cdot 4$ & & & & & C-R & & & $\mathrm{R}$ & A & A & & & & $\mathrm{R}$ & & & & & $\begin{array}{l}\text { Fish debris is } \\
\text { bright green. }\end{array}$ \\
\hline 50.1 & 3 & 5,6 & & & & & $\mathrm{R}$ & & & & $\mathrm{A}$ & $\mathrm{A}$ & & $\mathrm{R}$ & & $\mathrm{R}$ & & & & & \\
\hline 50.1 & 4 & 1 & & & & & $\mathrm{R}$ & & & & A & $\mathrm{A}$ & & $\mathrm{A}$ & & $\mathrm{R}$ & & & & & $\begin{array}{l}\text { Fish debris is } \\
\text { bright green. }\end{array}$ \\
\hline 51.0 & 1 & All & & $\mathrm{R}$ & $\mathrm{R}$ & & $\mathrm{C}$ & $\mathrm{R}$ & & & $\mathrm{A}$ & $\mathrm{A}$ & & $\mathrm{R}$ ? & & & & & & & $\begin{array}{l}\text { Fish debris is } \\
\text { bright green. }\end{array}$ \\
\hline
\end{tabular}


TABLE 2 - Continued

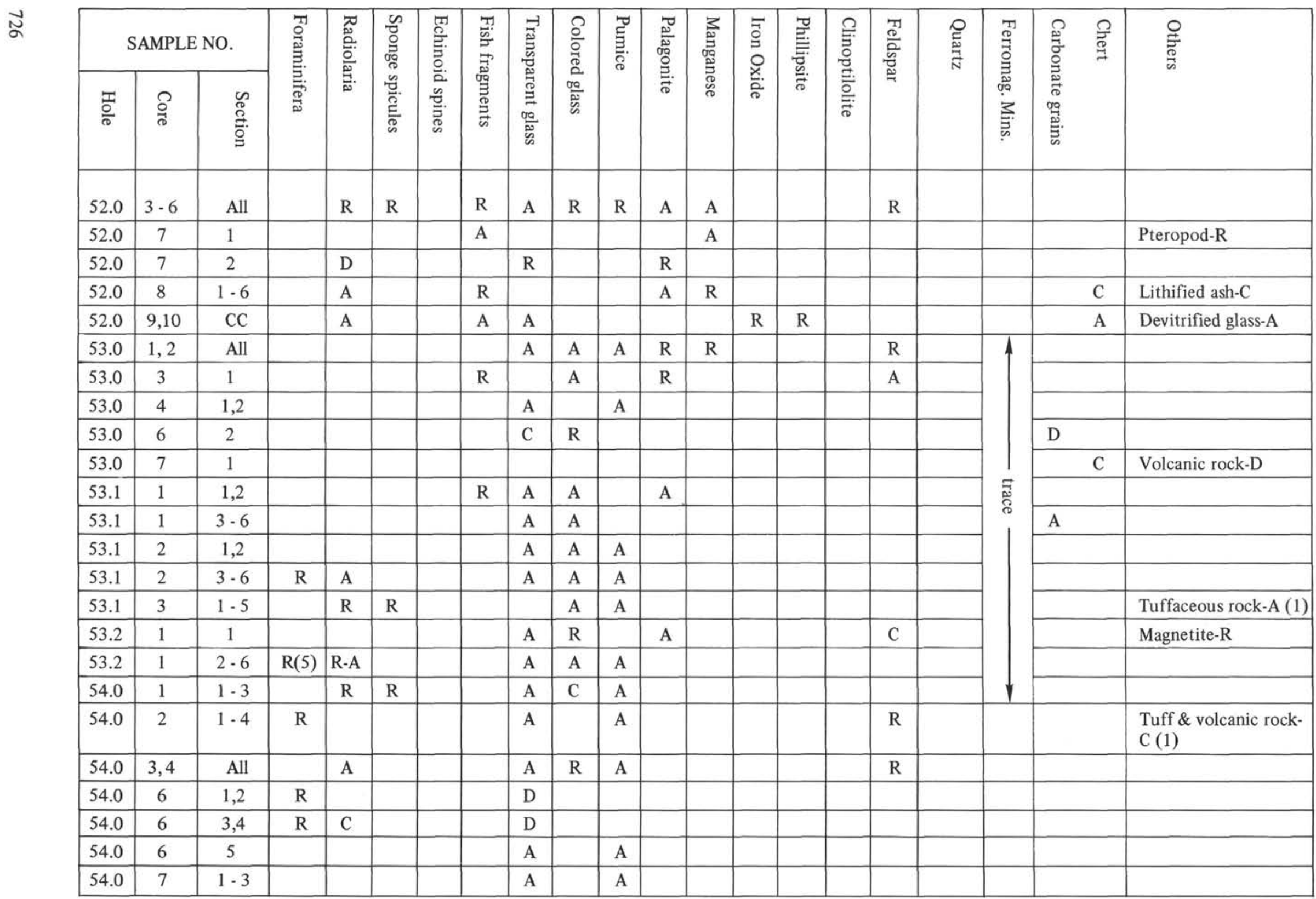


TABLE 2 - Continued

\begin{tabular}{|c|c|c|c|c|c|c|c|c|c|c|c|c|c|c|c|c|c|c|c|c|c|}
\hline \multicolumn{3}{|c|}{ SAMPLE NO. } & \multirow{2}{*}{ 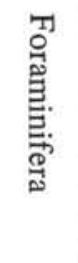 } & \multirow{2}{*}{ 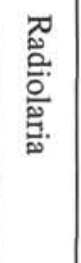 } & \multirow{2}{*}{ 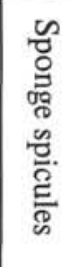 } & \multirow{2}{*}{ 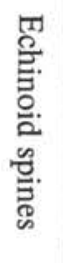 } & \multirow{2}{*}{ 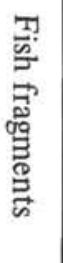 } & \multirow{2}{*}{ 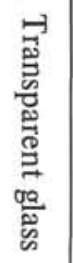 } & \multirow{2}{*}{ 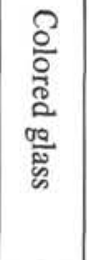 } & \multirow{2}{*}{ 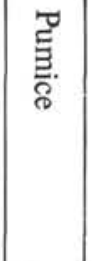 } & \multirow{2}{*}{ 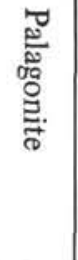 } & \multirow{2}{*}{ 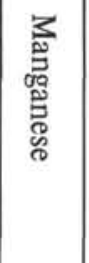 } & \multirow{2}{*}{ 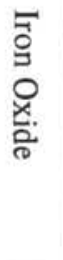 } & \multirow{2}{*}{ 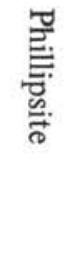 } & \multirow{2}{*}{ 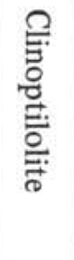 } & \multirow{2}{*}{ 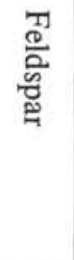 } & \multirow{2}{*}{ 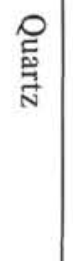 } & \multirow{2}{*}{ 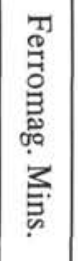 } & \multirow{2}{*}{ 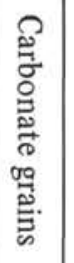 } & \multirow[t]{2}{*}{$\stackrel{\varrho}{\leftrightarrows}$} & \multirow{2}{*}{ 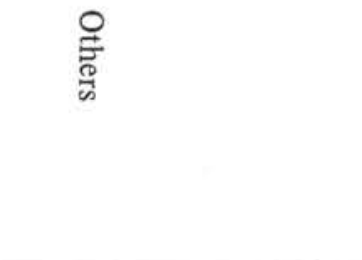 } \\
\hline 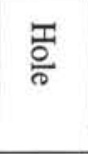 & @ & 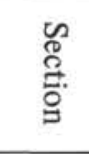 & & & & & & & & & & & & & & & & & & & \\
\hline 55.0 & $1 \cdot 5$ & All & $\mathrm{D}$ & $\mathrm{R}$ & $\mathrm{R}$ & & & $\mathrm{R}$ & & & $\mathrm{R}$ & & & & & $\mathrm{R}$ & & & & & \\
\hline 55.0 & $6-8$ & All & A & A & A & & $\mathrm{R}$ & $\mathrm{R}$ & & $\mathrm{R}$ & $\mathrm{R}$ & $\mathrm{R}$ & & & & & & & & & \\
\hline 55.0 & $9 \cdot 12$ & All & R-A & $\mathrm{A}$ & $\mathrm{A}$ & & & & & & & & & & & & & & & & Diatom-R \\
\hline 55.0 & 13 & 1 & $\mathrm{~A}$ & A & & & & $\mathrm{A}$ & A & & & & & & & & & & & & \\
\hline 55.0 & 13 & 3,5 & A & $\mathrm{A}$ & & & & & & & & & & & & & & & & & \\
\hline 55.0 & 14 & 1.6 & $\mathrm{~A}$ & $\mathrm{~A}$ & & $\mathrm{R}$ & & & & $\mathrm{R}$ & & $\mathrm{R}$ & & & & & & & & & \\
\hline 56 & $1-6$ & All & $\mathrm{D}$ & $\mathrm{R}$ & $\mathrm{R}$ & & $\mathrm{R}$ & & & & & & & & & & & & & & $\begin{array}{l}\text { Glass \& pumice-R } \\
\text { in Core } 6 \text { only. }\end{array}$ \\
\hline 56.2 & $7 \cdot 10$ & All & $\mathrm{D}$ & R-C & $\mathrm{R}-\mathrm{C}$ & $\mathrm{R}$ & & $\mathrm{R}$ & $\mathrm{R}$ & $\mathrm{R}$ & & & & & & & & & & & Diatom-R \\
\hline 57.1 & 1 & $1-6$ & $\mathrm{D}$ & R-C & $\mathrm{R}$ & $\mathrm{R}$ & & & $\mathrm{R}$ & & & $\mathrm{R}$ & & & & & & & & & \\
\hline 57.1 & 2,3 & All & $\mathrm{A}$ & $\mathrm{R}$ & $\mathrm{R}$ & & & & $\mathrm{A}$ & & & C-A & & & & $\mathrm{R}$ & & & & $\mathrm{R}$ & \\
\hline 57.1 & 4 & All & $\mathrm{A}$ & $\mathrm{R}-\mathrm{C}$ & & & & & A & R-C & & & & & & & & & & & \\
\hline 57.2 & 1 & All & $\mathrm{D}$ & & & & & & & & & & & & & & & & & & \\
\hline 58.1 & 1 & 1 & $\mathrm{D}$ & $\mathrm{C}$ & & & & & & & & & & & & & & & & & \\
\hline 58.1 & 1 & 2 & $\mathrm{D}$ & $\mathrm{C}$ & $\mathrm{R}$ & & & & & & $\mathrm{R}$ & & & & & & \multirow{2}{*}{\multicolumn{2}{|c|}{$\uparrow$}} & & & \\
\hline 58.1 & 1 & 3 & $\mathrm{D}$ & $\mathrm{C}$ & & & & & $\mathrm{R}$ & & & & & & & & & & & & \\
\hline 58.2 & 1 & 1 & & $\mathrm{~A}$ & A & & & $\mathrm{R}$ & $\mathrm{R}$ & & $\mathrm{A}$ & & & & & & \multirow{2}{*}{\multicolumn{2}{|c|}{ 苛 }} & & & $\begin{array}{l}\text { At } 60 \mathrm{~cm} \text {, chalk, foram, } \\
\text { and volcanic rocks } C\end{array}$ \\
\hline 58.2 & 1 & 3 & & $\mathrm{~A}$ & $\mathrm{~A}$ & & & $\mathrm{R}$ & $\mathrm{R}$ & $\mathrm{C}$ & & & & & & & & & & & \\
\hline 58.2 & 1 & 4 & A & $\mathrm{C}$ & $\mathrm{C}$ & & & & R-A & $\mathrm{A}$ & & & & & & & & 1 & & & \\
\hline 59.2 & 1,2 & All & & A & $\mathrm{A}$ & & & & & & $\mathrm{R}$ & & & & & & & & & & \\
\hline 59.2 & 3 & All & & A & $\mathrm{C}$ & & & & & $\mathrm{R}$ & R-C & R-C & & & & & & & & & \\
\hline 59.2 & 5 & 1 & & & & & $\mathrm{R}$ & & & & $\mathrm{R}$ & $\mathrm{R}$ & & $\mathrm{R}$ & D & & & & & & \\
\hline 59.2 & 6 & 2 & & $\mathrm{~A}$ & $\mathrm{R}$ & & & $\mathrm{R}$ & & & $\mathrm{A}$ & $\mathrm{R}$ & & $\mathrm{R}$ & $\mathrm{A}$ & & & & & C & \\
\hline 60.0 & 3 & 1,2 & & & & & & $\mathrm{R}$ & A & A & & & & & & & & & & & \\
\hline 60.0 & 4 & 1,2 & & & & & & $\mathrm{~A}$ & $\mathrm{~A}$ & & & & & & & & & 劳 & & & \\
\hline
\end{tabular}


TABLE 2 - Continued

$\underset{\infty}{\mathbb{N}}$

\begin{tabular}{|c|c|c|c|c|c|c|c|c|c|c|c|c|c|c|c|c|c|c|c|c|c|}
\hline \multicolumn{3}{|c|}{ SAMPLE NO. } & \multirow{2}{*}{ 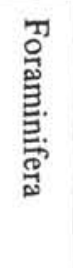 } & \multirow{2}{*}{ 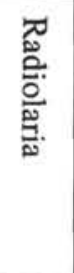 } & \multirow{2}{*}{ 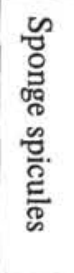 } & \multirow{2}{*}{ 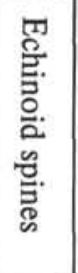 } & \multirow{2}{*}{ 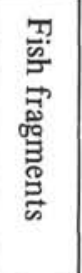 } & \multirow{2}{*}{ 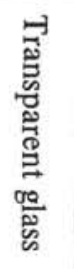 } & \multirow{2}{*}{ 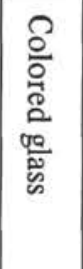 } & \multirow{2}{*}{ 菅. } & \multirow{2}{*}{$\begin{array}{l}\text { 总 } \\
\text { o. } \\
\text { 总. }\end{array}$} & \multirow{2}{*}{ 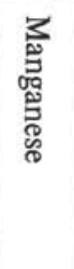 } & \multirow{2}{*}{ 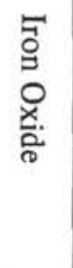 } & \multirow{2}{*}{ 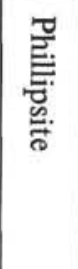 } & \multirow{2}{*}{ 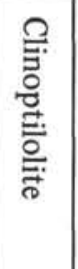 } & \multirow{2}{*}{ 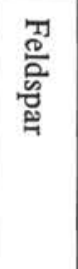 } & \multirow{2}{*}{ 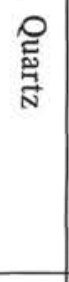 } & \multirow{2}{*}{ 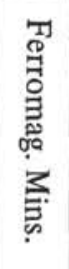 } & \multirow{2}{*}{ 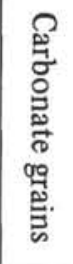 } & \multirow[t]{2}{*}{$\begin{array}{l}\stackrel{?}{0} \\
\stackrel{\emptyset}{q}\end{array}$} & \multirow{2}{*}{ 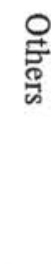 } \\
\hline$\frac{\pi}{\frac{T}{\sigma}}$ & $\oint_{\varnothing}$ & 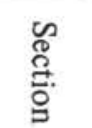 & & & & & & & & & & & & & & & & & & & \\
\hline 60.0 & 5 & 1,2 & & & & & & D & & & & & & & & & & 1 & & & \\
\hline 60.0 & 6 & 1.4 & & & & & & $\mathrm{C}$ & D & & & & & & & & & & & & \\
\hline 60.0 & 6 & 6 & D & & & & & & & & & & & & & & & $\Rightarrow$ & & & \\
\hline 60.0 & 7 & 1,2 & & & & & & D & $\mathrm{R}$ & & & & & & & & & గ్ర & & & \\
\hline 60.0 & 8 & 2 & D & $\mathrm{R}$ & $\mathrm{R}$ & & & $\mathrm{R}$ & $R$ & & & & & & & & & & & & \\
\hline 60.0 & 9 & 3 & & & & & & $R$ & D & & & & & & & $\mathrm{C}$ & & 1 & & & \\
\hline
\end{tabular}

$\mathrm{D}=$ Dominant $>75$ per cent

$A=$ Abundant $75-15$ per cent

$$
\begin{aligned}
& C=15-5 \text { per cent } \\
& R=<5 \text { per cent }
\end{aligned}
$$


faecel pellet origin. Palagonite and fish fragments are also common. Transparent volcanic glass and indeterminate siliceous grains occur rarely.

\section{Site 46}

The dominant constituent in Hole 46.0 is poorly preserved, robust Radiolaria. Chert, lithified ash, and manganese are also common throughout the sand fraction in this hole. Fossil relicts are visible in the chert fragments. Fish fragments and sponge spicules occur rarely.

\section{Site 47}

The sand fraction from the one core in Hole 47.0 (Pleistocene) predominantly comprises foraminifera with less common sponge spicules, echinoid spines, and Radiolaria. Small quantities of chalk (apparently from inside foraminifera tests) also occur. Abundant transparent glass shards occur in Section 2. In Section 5, Radiolaria are as common as foraminifera. In Hole 47.1 (Paleocene) and in Cores 1 through 6 of Hole 47.2 (Pleistocene-Miocene), foraminifera comprise the main constituent of the sand fraction. Minor constituents include sponge spicules, echinoid spines, fish fragments, Radiolaria, chalk, and manganese. In a few places Radiolaria are as abundant as foraminifera. In Section 4, Core 3 , faecel pellets and ostracods were noted.

In Cores 7 through 10 of Hole 47.2 foraminifera again predominate. Minor constituents are very rare and when present are similar to those in the upper part of the hole, however, in Section 1 of Core 7 several small multiple twinned, pale brown phillipsite crystals occur. A major unconformity between Upper Miocene and Lower Eocene sediments occurs between Sections 1 and 2 of Core 7 in Hole 47.2. Also rarely present in Section 1, Core 7 are pale green volcanic glass (?) fragments. In Core 9 fish fragments become more common. In a few places quartz, manganese, iron oxide and yellow-green pumice occur rarely.

In Core 11, the sand fraction is comprised predominantly of very small planktonic foraminifera with only rare volcanic glass and Inoceramus prisms.

In Sections 1 and 2 of Core 12 angular fragments of transparent to pale yellow chert are as common as foraminifera; a few red fragments of chert were also seen. In Section 2, Core 12, manganese is common, palagonite and Inoceramus prisms are rare. Sections 3 through 6 of Core 12 and all of Core 13 consist almost entirely of foraminifera, but in Sections 4 and 5 of Core 12 chert and palagonite are common.

\section{Site 48}

In Hole 48.1 the sand fraction is comprised predominantly of foraminifera and palagonite (?) with minor amounts of Radiolaria, echinoid spines, sponge spicules, thin transparent volcanic glass and feldspar (?).
In Hole 48.2 , Core 1 , Sections 1 to 5 , foraminifera are abundant together with small amounts of chalk. Minor constituents include Radiolaria, manganese, thin transparent glass, fish fragments, echinoid spines and sponge spicules. In Sections 3 and 4, and to a lesser extent in Section 5, radiating clusters of pale orange-brown phillipsite crystals occur commonly. Sediments here are of Upper Miocene age and unconformably overlie the Maestrichtian sediments of Section 6.

Cores 2 and 3 of Hole 48.2 only contain Cretaceous foraminifera in the sand fraction.

\section{Site 49}

In Core 1 of Hole 49.0 Pleistocene sediments contain abundant palagonite, manganese and thin transparent glass in the sand fraction. Minor quantities of Radiolaria, fish fragments and feldspar occur. Reworked foraminifera occur rarely. In Sections 1 and 6, small quantities of phillipsite are present.

In Core 2, sediments of Tithonian (?) age contain manganese, chalk and foraminifera in abundance. Smaller amounts of Radiolaria, fish fragments, transparent glass, palagonite and phillipsite occur.

In Hole 49.1, Core 1, Section 1 thin transparent volcanic glass, palagonite and manganese are common. In Sections 2 and 3 phillipsite is abundant and fish fragments become common; feldspar occurs rarely. Note that the phillipsite of Sections 2 and 3 occurs in Pleistocene sediments which lie unconformably on Tithonian sediments in the lower part of Core 1 . Sections 4 through 6 of Core 1 mainly comprise manganese and palagonite with chert and chalk fragments becoming common near the base. Poorly preserved Radiolaria and foraminifera, fish fragments and phillipsite (possibly contamination from up the hole) occur in minor amounts.

The sand fraction in Core 2 of Hole 49.1 (Tithonian? age) is similar to that in the lower part of Core 1 with the exception that phillipsite is lacking.

\section{Site 50}

In Core 2 of Hole 50.0 Tithonian (?) sediments contain poorly preserved Radiolaria in abundance with a few pebbles of chert, chalk, manganese and limonite. Minor amounts of feldspar and yellow volcanic glass are also present.

In Cores 1 and 2 of Hole 50.1 of Pleistocene age, there are abundant Radiolaria and diatoms of foraminifera (depending on the sediment type) with common thin transparent glass and pale to dark brown pumice; smaller amounts of palagonite, sponge spicules, echinoid spines and manganese also occur. Small (fine sand size) feldspar and amphibole grains are rare. 
Core 3 mainly comprises manganese and palagonite with numerous bright yellow-green fish fragments in places. Minor amounts of white semi-transparent fish fragments feldspar and, in the base of Core 3 , phillipsite also occur.

In Core 4 (Jurassic to Cretaceous ?) the sand fraction is similar to Core 3 , but here phillipsite becomes abundant.

\section{Site 51}

Miocene sediments in Hole 51.0 contain abundant manganese and common palagonite. Minor quantities of thin transparent volcanic glass, sponge spicules, fish fragments, Radiolaria and phillipsite occur. In Section 4 abundant bright yellow-green fish fragments occur.

In Hole 51.1, Core 1, Radiolaria with some diatoms predominate with smaller amounts of palagonite, or pale brown pumice. In Section 6, thin transparent volcanic glass becomes abundant. Sponge spicules, colored volcanic glass and fish fragments occur rarely.

In Core 2, poorly preserved Radiolaria predominate, fish fragments are common, and minor amounts of manganese, thin transparent volcanic glass, chalk, phillipsite (?) and chert (?) are present.

\section{Site 52}

Cores 1 and 2 of Hole 52.0, comprising sediments of Quaternary to Tertiary age, have as the main constituents of their sand fraction thin transparent volcanic glass, pumice ranging in color from transparent to dark brown-black, and palagonite. Less commonly, red and olive-green glass fragments occur. Manganese is quite common in places, and fish fragments, spicules and Radiolaria are also present.

Cores 3 through 6 consist mainly of palagonite and thin transparent glass, with manganese becoming predominant from Core 4 down. Minor constituents in these cores are similar to those above.

In Core 7 , the sand fraction is mainly manganese and fish fragments with less common palagonite, thin transparent glass, and rarely pteropod fragments. In the lower part of Section 2 altered Radiolaria predominate.

In Core 8 (Cretaceous ?) altered Radiolaria and palagonite are abundant. Pebble-sized ash and chert fragments are common in the upper part. Minor constituents include fish fragments and manganese.

In core catcher samples from Cores 9 and 10 (Cretaceous) the sand fraction mainly comprises fresh transparent glass, altered glass, palagonite, clay granules (after glass?), fish debris and otoliths, silicified Radiolaria, and chert fragments. Minor amounts of iron oxide, opaque minerals, phillipsite and silicified rock fragments were also seen.

\section{Site 53}

The sand fraction in Hole 53.0, Core 1, is mainly thin transparent volcanic glass with abundant yellow, olivegreen, brown and black glass and pumice. In the lower part of Core 1, and all of Core 2, the colored varieties of pumice predominate. Manganese, red (hematitic ?) glass, palagonite and feldspar occur rarely. In Core 3 red and green glass and feldspar are common, together with lesser amounts of palagonite and fish fragments. In Core 4 the sand is fine-grained and very well sorted; it comprises transparent glass and all colored varieties of pumice, Pinkish carbonate grains predominate in Core 6; some transparent and red glass is also present. Green-brown volcanic rock fragments, and less commonly chert, predominate in Core 7 .

The upper part of Core 1 in Hole 53.1 comprises palagonite, transparent and colored glass, with rare fish fragments. From Section 3 down in Core 1 abundant yellowish-white carbonate grains and tubules are common, together with all varieties of transparent and colored glass. The upper part of Core 2 comprises transparent and colored varieties of glass and pumice; and, in Sections 3 through 6 from this core, Radiolaria and, in a few places, foraminifera are also common. In Core 3 dark gray, olive-gray and black pumice and glass are common. In Section 1 of this core abundant pebbles of pumice and tuffaceous rock occur; Radiolaria and sponge spicules are rare.

In Hole 53.2, Core 1, Section 1, the sand fraction mainly comprises palagonite and feldspar, with less common olive-green glass and magnetite octahedra. A few of the fledspar grains are zoned and of intermediate composition. Sections 2 through 6 contain varying amounts of Radiolaria in addition to glass and pumice; and in Section 5 there are large agglutinating benthonic foraminifera.

\section{Site 54}

In Core 1 transparent glass and pumice predominate with less common green, red and yellow glass, and rarely Radiolaria and spicules. Core 2 mainly comprises thin transparent glass (andesitic) and pumice; feldspar (intermediate composition) and foraminifera occur rarely. In Section 1 of this core pebbles of pumice, tuff and limonitised volcanic (?) rock occur. Cores 3 and 4 contain similar sand grains to those in Core 2, together with numerous Radiolaria. Core 6 comprises thin transparent glass, colorless pumice, with foraminifera and Radiolaria in places. In Core 7 mostly colorless to black pumice of granule size occur together with finer transparent glass. The volcanic glass at this site commonly contains a few to abundant vesicles (glass looks almost opaque).

\section{Site 55}

Cores 1 through 5 consist almost entirely of planktonic foraminifera with very minor amounts of fish fragments, 
Radiolaria, sponge spicules, feldspar, glass, and palagonite in a few places.

In Cores 6 through 8 foraminifera again predominate, but Radiolaria and spicules are common. A few grains of transparent volcanic glass, palagonite, pumice, fish fragments and manganese occur.

Cores 9 through Section 3, Core 12 contain abundant Radiolaria with spicules, and minor amounts of transparent volcanic glass and diatoms. Foraminifera are common from Core 11 down. In the lower part of Core 12 , foraminifera are more abundant than the siliceous fossils.

In Section 1 (4 centimeters depth) of Core 13, the sand fraction comprises abundant transparent to pale brown volcanic glass shards or fragments with tubular vesicles, and foraminifera. Radiolaria are also common. Glass is lacking in the remainder of Core 13, but becomes common again in Core 14. Radiolaria are quite common, and echinoid spines, pumice and manganese occur rarely.

\section{Site 56}

The sand fraction from Hole 56.2 predominantly comprises planktonic foraminifera throughout. Minor amounts of Radiolaria, sponge spicules and, in places, fish fragments were seen; the siliceous fossils generally become more common in the lower part of the hole (Cores 7 through 10). Also present in the lower part (Cores 6 through 10) of this hole are small amounts of transparent and colored volcanic glass and pumice. Benthonic foraminifera, echinoid spines and diatoms occur rarely.

\section{Site 57}

The sand fraction in Hole 57.1 comprises very small foraminifera with a few fish fragments. Near the top of this core, minor amounts of olive-brown glass, spicules, echinoid spines and manganese occur. Radiolaria are common in Sections 1 and 4. In Core 2, foraminifera predominate and manganese is common. Minor constituents are similar to Core 1 . In Core 3 foraminifera are abundant or rare, depending on the sediment type. Vesicular pale brown to brown glass is common throughout this core. Manganese is common in Section 1; Radiolaria and feldspar are rare.

The sand fraction in Core 4 is similar to that of Core 3 , with the addition of abundant black pumice and, occasionally, lithified chalk fragments. The foraminifera in this core are very small.

In Hole 57.2 the sand fraction is entirely foraminifera.

\section{Site 58}

The sand fraction in Hole 58.1, Core 1, mainly comprises foraminifera with some Radiolaria. Palagonite and spicules occur in Section 2, and in Section 3 small amounts of yellow, orange, red, and brown vesicular glass are present.

In Hole 58.2, Core 1, Radiolaria, radiolarian spines, and spicules are abundant. Yellow palagonite and olive brown glass are rare. A few reworked foraminifera are also present. In a few places (Section 1, 60 centimeters) pebbles of hard chalk and volcanic rocks are present; the sand-size material here is predominantly foraminifera, Radiolaria, and spicules with less common palagonite, transparent and colored glass, and pumice. In Section 3 of this core Radiolaria, radiolarian spines, and spicules are abundant, yellow-orange palagonite and brown-black pumice are common; transparent and colored glass are rare. In Section 4 foraminifera and orange-brown-black pumice predominate, with Radiolaria and spicules being common. At 130 centimeters depth in Section 4 volcanic glass is nearly all yellowbrown to dark brown.

\section{Site 59}

The sand fraction in Hole 59.2, Cores 1 and 2, mainly comprises Radiolaria and spicules, with smaller amounts of palagonite. In Core 3 black pumice, manganese, palagonite (?) and fish fragments occur in addition to the main constituents of Core 1 .

In Cores 5 and 6 the main constituents are radiating clusters of the zeolite mineral clinoptilolite, palagonite, and Radiolaria. Pebble-sized chert fragments occur in Section 2 of Core 6. Manganese, fish fragments, spicules, transparent glass and phillipsite occur less commonly.

\section{Site 60}

The sand fraction in Hole 60.0 is nearly all volcanic glass of varying types. The main variations are as follows:

Core 3-2, $4 \mathrm{~cm}$ : Transparent and yellow-brown glass, and white to brown pumice dominant:

Core 3-2, $121 \mathrm{~cm}$ : Very well sorted, sub-angular, equidimensional brown to black, with some yellow, volcanic glass, dominant:

Core 4-1, $40 \mathrm{~cm}$ : Predominantly thin transparent and black glass:

Core 4-2, $145 \mathrm{~cm}$ : Well sorted, thin, transparent and very pale brown elongate glass shards:

Core 5-2, $20 \mathrm{~cm}$ : Entirely thin transparent glass: 
Core 6-1, $79 \mathrm{~cm}$ : Sorted, sub-angular, pale brown to black, with some transparent, glass, red glass occurs rarely:

Core 6-3, $7 \mathrm{~cm}$ : Transparent red and brown glass predominant; spicules occur rarely:

Core 6-6:

Core $7-1,2 \mathrm{~cm}$ :
The sand fraction here is entirely foraminifera:

Predominantly transparent glass with minor amounts of brown to black grass, the glass fragments are elongate and sorted:

Core 8-2:

Core 9-3

Foraminifera predominate and Radiolaria, spicules and glass occur rarely:

The coarse sand is all yellow, pale brown, and black glass; the fine sand is mainly transparent feldspar with some pale brown to yellow glass. Red and olive-brown glass also occur rarely. The sand grains in this core are all equidimensional.

\section{PLATE 1}

Electron Scan Photographs of Sand Fraction of Miocene to Middle

Pleistocene Brown Clay (Courtesy of S. A. Kling, Cities Oil Service)

Figure 1 Sample 52.0-1-1, $22 \mathrm{~cm}$ (X 100):

Bubble shards of volcanic glass, pumice, Radiolaria, sponge spicules, and one crystal of phillipsite.

Figure 2 Sample 52.0-1-1, $22 \mathrm{~cm}(\times 100)$ :

Bubble shards of glass and pumice.

Figure 3 Sample 52.0-1-1, $77 \mathrm{~cm}(\times 100)$ :

Pumice, glass with tubularvesicles, bubble shards, and feldspar fragments.

Figure 4 Sample 52.0-3-5, $1 \mathrm{~cm}(\times 100)$ :

Pumice and feldspar grains with rare Radiolaria fragments. 

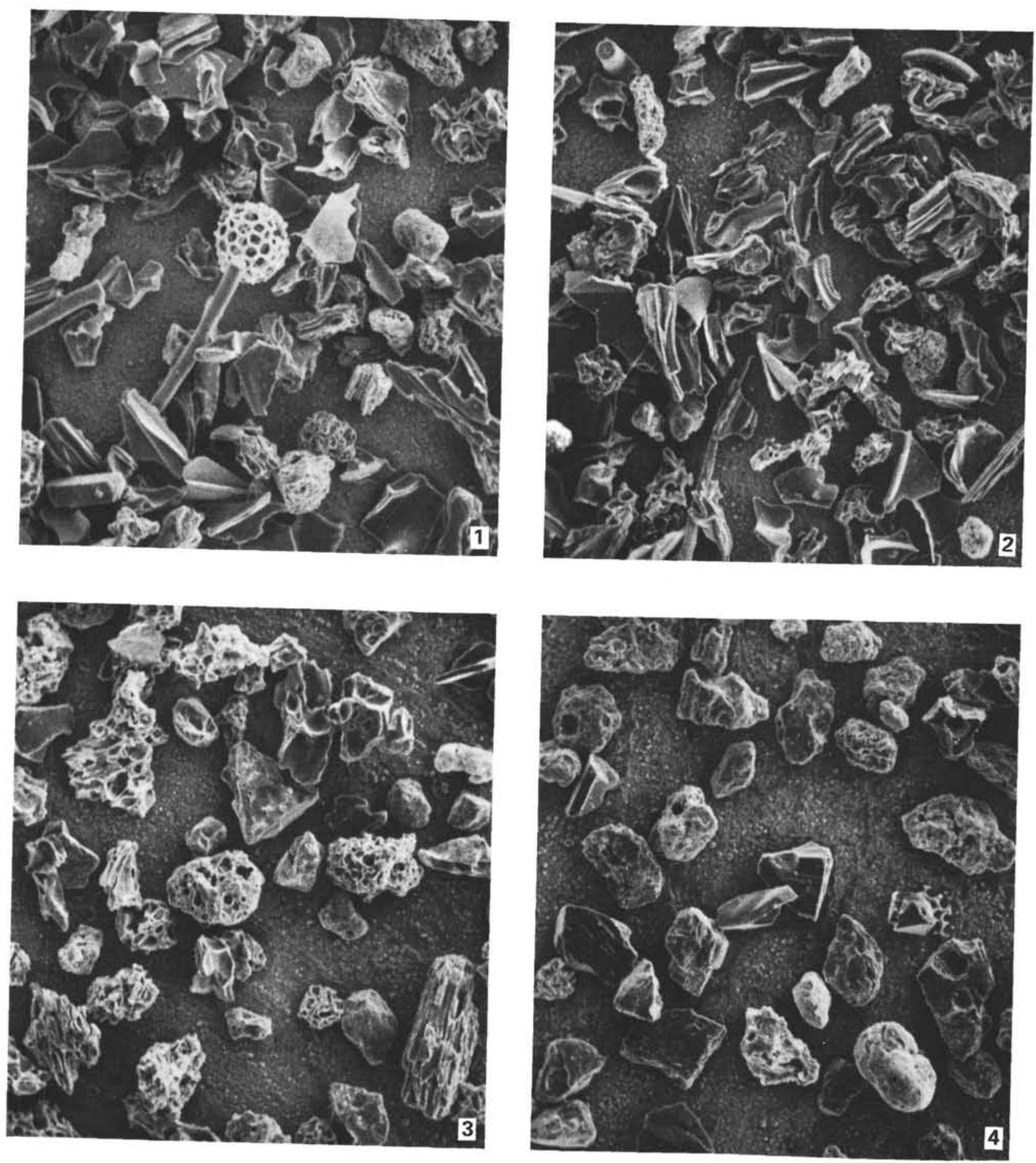


\section{PLATE 2}

Electron Scan Photographs of Sand Fraction of Volcanic Ash (Courtesy S. A. Kling, Cities Oil Service)

Figure 1 Sample 53.1-2-1, $145 \mathrm{~cm}(\times 100)$ :

Late Miocene pumice fragment.

Figure 2 Sample 53.1-2-1, $145 \mathrm{~cm}$ ( $\times 1000)$ :

Enlargement of portion of pumice fragment shown in Figure 1.

Figure 3 Sample 54.0-6-1, $145 \mathrm{~cm}(\times 100)$ :

Early Middle Miocene.

Mostly glass fragments with tubular vesicles, some bubble shards and pumice, rare sponge spicules.

Figure 4 Sample 60.0-3-2, $121 \mathrm{~cm}$ (X 100):

Middle Middle Miocene.

Well-sorted water worn grains of pumice and volcanic glass. 

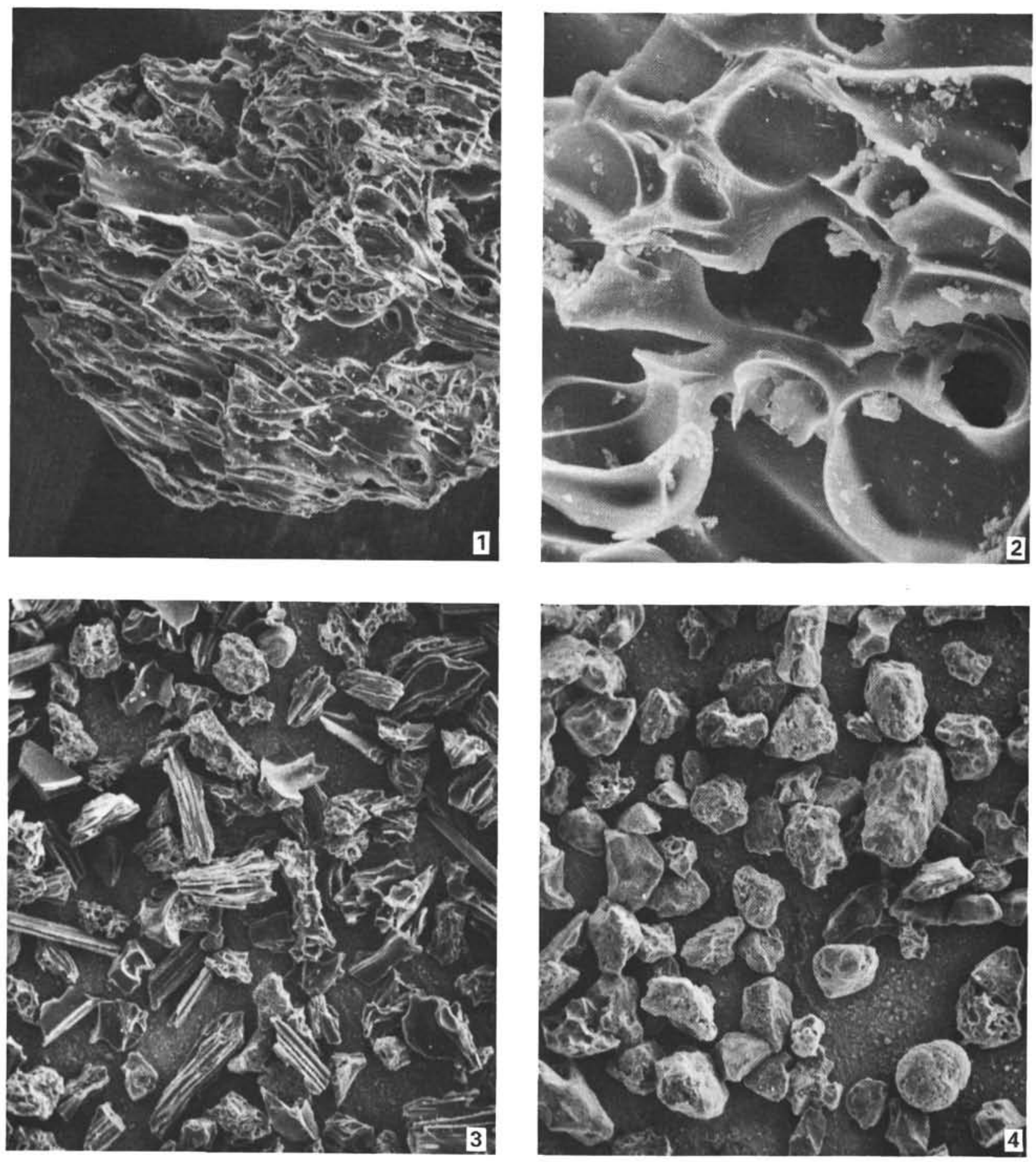


\section{PLATE 3}

Electron Scan Photographs of Sand Fraction from Variety of Sediment Types (Courtesy S. A. Kling, Cities Oil Service)

Figure $1 \quad$ Sample 49.1-1.2, $119 \mathrm{~cm}(\times 100)$ :

Zeolitic clay from interval of Pleistocene/late Jurassic or early Cretaceous unconformity on Shatsky Rise. Abundant phillipsite crystals with a few grains of pumice, feldspar and fish fragments.

Figures 2 and 3 Sample 59.2-5-1, $82 \mathrm{~cm}(\times 100)$ :

Late Eocene to early Oligocene zeolitic clay.

Mostly phillipsite crystals and aggregates of clinoptilolite with less common Radiolaria and fish fragments.

Figure 4

Sample 53.1-2-1, $145 \mathrm{~cm}(\times 100)$ :

Late Miocene volcanic ash.

Mostly pumice, bubble shards and rare feldspar grains. 

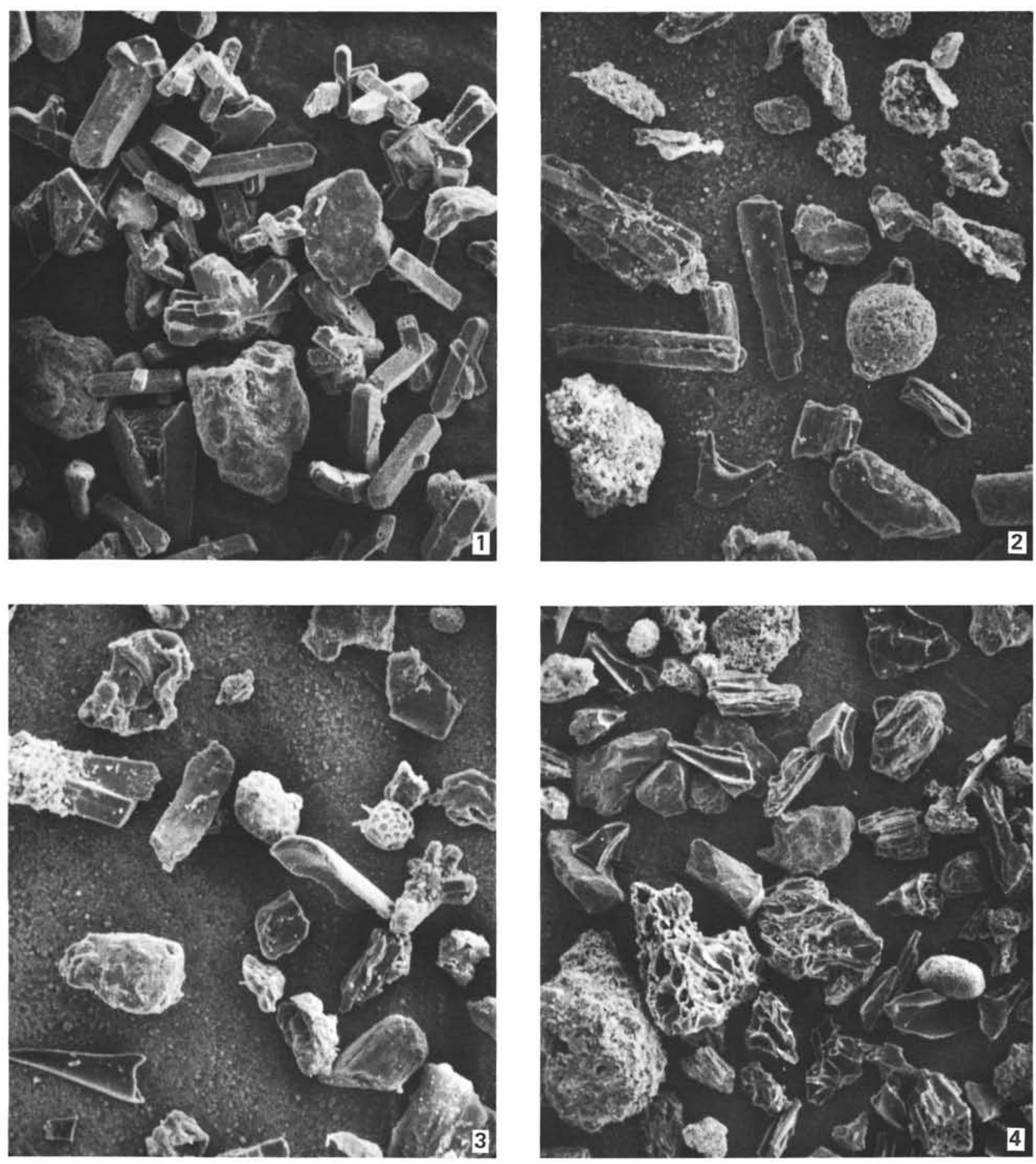